\title{
Über den Einfluß der Nahrungsaufnahme auf die Ausscheidung der Harnsäure bei Arthritis urica.
}

\author{
Von \\ Franz Soetbeer. \\ Mit vier Tafeln. \\ (Aus dem Laboratorium der Kinderklinik zu Heidelberg und dem Eppendorfer \\ Krankenhause zu Hamburg.) \\ (Der Redaktion zugegangen am 11. September 1903.)
}

Es ist möglich, daß auf guten Fundamenten grade aufgebaute Hypothesen als Untergrund für den Aufbau neuer Fragestellungen die Forschung auf einem Gebiete glänzend fördern. Wir haben das wieder bei der Immunitätsforschung erlebt. Bei der Gichtforschung haben die zahllosen hypothetischen Krankheitstheorien nur hemmend gewirkt, haben die dürftigsten tatsächlichen Kenntnisse mit einem blendenden Gedankenflitter verhüllt. Sind die Fundamente schlecht, so gibt es für die Vertiefung der Erkenntnis wohl keine getährlicheren Hemmnisse, als solche hohlen Hypothesen, die den Abgrund unserer Unkenntnis trügerisch überbrücken. Sie lassen eine Frage abgeschlossen oder abgeschlossener erscheinen, als sie in Wirklichkeit ist, und das ist das größte Hemmnis für ihre wirkliche Erforschung.

Doch neben den Theorien ist auf dem Gebiete der Gichtforschung eine Fülle von Tatsachen produziert, deren Bewertung eine verschiedene ist. Stellen wir die besten zusammen, so müssen wir ein objektives Bild unserer jetzigen Kenntnis dieser Krankheit erhalten.

Was wissen wir von der Gicht? 
1. Von ihren Entstehungsursachen wissen wir wenig Sicheres, als einzig gut fundiertes ätiologisches Moment ist. außer der Heredität das Blei ${ }^{1}$ ) zu betrachten.

2. Von ihrem Verlauf, ihren klinischen Symptomen, ihren anatomischen Äquivalenten oder Begleiterscheinungen haben wir gute, von Meistern der Beobachtung und Darstellung erforschte und aufgestellte Bilder.

Wir kennen nur nicht den inneren Zusammenhang der Symptome. Wir suchen vergebens nach der einheitlichen Ursache, die im Organismus solch zahlreiche, verschiedenartige, anatomische Veränderungen an allen Organen bewirkt.

Man sah die Urate in den Gelenken und glaubte hier die Ursache, die materia peccans des ganzen Prozesses, gefunden zu haben, die Harnsäure.

Man erhob und beantwortete die Frage: "Welche Rolle spielt die Harnsäure in der Gichtpathologie?» Man beantwortete die Frage nach der pathologischen Wirksamkeit eines Stoffes, dessen physiologische Rolle im Säugetierorganismus uns noch immer ein Buch mit sieben Siegeln ist.

Eine immer wieder kraftvoll einsetzende unermüdliche Arbeit unserer besten Physiologen und Chemiker sucht bedachtsam, schrittweise den Rätseln des Harnsäurestoffwechsels näher zu treten. Sie haben die chemische Konstitution festgelegt und das physikalisch chemische Verhalten zu fixieren versucht. Sie haben die der Harnsäure chemisch verwandten Stoffe gefunden und dargestellt und den Übergang von einem in den andern experimentell nachgewiesen. Mit der Entdeckung Kossels, daß alle diese Stoffe aus den Nucleinen durch Spaltung und Oxydation darstellbar sind, hat sich von den Nucleinen des Zellleibes eine lange Kette von Substanzen bis zur Harnsäure herunter gebildet, der die unermüdliche, chemisch-physiologische Forschung immer neue Glieder einzureihen bemüht ist.

Alle diese Stoffe können auch im Organismus im intermediären Stoffwechsel der Nucleine eine Rolle spielen, ob sie es wirklich tun, davon wissen wir bis jetzt wenig oder nichts.

1) Lüthje, Zeitschr. f. klin: Med., Bd. 29, S. 266. 
Der Mangel eines geeigneten Versuchstiers für Harnsäurestoffwechselversuche macht sich hier am meisten geltend, nachdem man den Hund als solches, ebenso wie das Kaninchen nun wohl endgültig beiseite getan hat. Die einzig gültigen Resultate sind die am Menschen gefundenen.

Minkowski1) hat nach Einverleibung von Hypoxanthin eine Vermehrung der Harnsäure des Harns gefunden. Damit ist gezeigt, daß Aufnahme von Hypoxanthin einen Einfluß auf die Harnsäureausscheidung hat (dasselbe bestätigen Burian²) und Schur), der Beweis für die Stellung des Hypoxanthins als «Vorstufe» der Harnsäure ist meines Erachtens noch nicht erbracht. Noch viel weniger für andere Stoffe wie Guanin von Loewi ${ }^{3}$ ) und Xanthin von Burian und Schur $;^{4}$ ) bei diesen Stoffen ist nicht einmal die Harnsäurevermehrung im Harn einwandfrei erwiesen. Teilweise im Gegensatz zu diesen Autoren wollen Krüger und Schmid ${ }^{5}$ ) nach Hypoxanthin, Adenin, Xanthin eine sichere, nach Guanin eine bedingte Vermehrung der Harnsäureausscheidung beim Menschen beobachtet haben. Leider vermissen wir die Angabe der Herkunft der verfütterten Stoffe und ihre Analyse. - Es kommt bei dem, was die Autoren beweisen wollen, Steigerung der Harnsäureausscheidung bei Verfütterung sfreier Purinbasen alles auf ihre Analysenreinheit an. Es wäre sehr wünschenswert, wenn das Material vorhanden wäre, daß noch nachträglich die Analysenzahlen der Stoffe veröffentlicht würden.

Der Pathologe muß sich klar darüber werden, daß nur die Erkenntnis der physiologischen Stellung der Harnsäure im Stoffwechsel ihn befähigen wird, ihr in der Pathologie der Gicht den richtigen Platz anzuweisen.

Will der Kliniker die ihm rätselhafte Krankheit in ihrem Wesen ergründen helfen, muß er entweder sich diesen Forschungen der Physiologie anschließen (His, Minkowski) oder er muß den Boden vorbereiten, um später die exakten Beobachtungen des Physiologen mit gleich exakten Resultaten der Krankenbeobachtung verbinden zu können. (Weintraud, His, Magnus Levy.)

1) Minkowsky, Arch. f. exp. Path., Bd. 41, S. 406.

$\left.{ }^{2}\right)$ Burian und Schur, Pflügers Archiv, 1900, Bd. 80, S. 315; Guanin S. 317.

3) Loewi, Arch. f. exp. Path., Bd. 45, S. 174.

$\left.{ }^{4}\right)$ Burian und Schur, Pflügers Arch., 1901, Bd. 87, S. 328.

5) Krüger und Schmid, Diese Zeitschr., Bd. XXXIV, S. 549 ff. 
Beide Gruppen arbeiten auf dasselbe Ziel von verschiedenen Seiten.

Wie weit sind die Kliniker? Ist die klinische Rolle der fertigen Harnsäure bei der Gicht schon völlig geklärt? Kennen wir auch nur die Gesetze eines parallelen Auftretens von klinischen Symptomen der Gicht mit Harnsäureausscheidungsstörungen? Wir können nur die letzteren beobachten, weil nur dafür die sichere Methode vorliegt.

Was haben die Beobachtungen am Krankenbett über das Verhalten der Harñsäureausscheidung bisher gezeitigt? Eine Fülle der widersprechendsten Beobachtungen von Garrod bis auf Kaufmann und Mohr.

Wir wollen hier nicht eine Aufzählung und Würdigung dieser Arbeiten einschalten, das ist die Aufgabe umfangreicher Monographien, die noch zuletzt glänzend gelöst ist in dem vorzüglichen Buch von Minkowski. ${ }^{1}$ ) Wir wollen aus der Fülle des Stoffes nur diejenigen herausgreifen, die uns gedient haben als Unterbau für die eigene Fragestellung.

$\mathrm{His}^{2}$ ) und Magnus Levy ${ }^{3}$ ) haben die breitesten und eingehendsten Beobachtungen über die Harnsäureausscheidung und ihr Verhalten zu den andern Symptomen der Gicht angestellt.

Sie konstatierten übereinstimmend, dass im Anfall und nach dem Anfall eine Zunahme der 24stündigen Harnsäureausscheidung stattfindet. Magnus Levy begnügte sich nicht, diese Tatsache zu konstatieren, sondern er erstreckte seine Beobachtung auf den Harnsäuregehalt des Blutes, um das Vorhandensein eines Parallelismus des Ansteigens und Fallens der Harnsäure im Harn und im Blut zu konstatieren. Eine konstante Vermehrung der Harnsäure im Blut entsprechend der Vermehrung im Harn konnte jedoch von Magnus Levy nicht konstatiert werden.

Wie sind die beiden Untersuchungsresultate nach unserer heutigen Kenntnis zu beurteilen?

Die Vermehrung der Harnsäure im Harn im Anfall, wohl verstanden, bei der von den Autoren aufgeführten gemischten

1) Minkowski, Die Gicht, Wien, 1903.

2) $\mathrm{His}$, Arch. f. klin. Med., Bd. 65, S. 156.

3) Magnus Levy, Zeitschr. f. klin. Med., Bd. 36, S. 353. 
Nahrung, ist eine Tatsache, mit der die Gichtpathologie stets. zu rechnen haben wird.

Die früheren Beobachtungen über die Harnsäuremengen im Blut sind seit der Arbeit Gotos ${ }^{1}$ ) in Kossels Laboratorium hinfällig geworden. Minkowski2) hat anscheinend ähnliche Beobachtungen mit gleichem Erfolg angestellt, wir vermissen jedoch nähere Angaben in der Literatur. Die klinischen Forschungsresultate über Harnsäure im Blut und Geweben sind durch die Entdeckung von der Nichtfällbarkeit der Harnsäure bei Gegenwart von Nuclein- und Thyminsäure vorläufig als annulliert zu betrachten. Wir wissen augenblicklich von dem wirklichen Gehalt an Harnsäure sowohl im normalen wie gichtischen Blut nichts. Wir können uns nach der Gotoschen Entdeckung kein Bild, keine Vorstellung mehr machen von dem Gehalt der Gewebe an Harnsäure, mit Ausnahme der Gelenke, in denen wir sie als harnsaure Salze auskrystallisiert vorfinden.

Auf Grund von Gotos Beobachtung nimmt Minkowski hypothetisch an, daß im normalen Gewebe die Harnsäure an Nucleinsäure gebunden ist, oder an irgend ein anderes Spaltungsprodukt des Nucleins mit gleich lösenden Eigenschaften. Es. muß so natürlich nach den vorhandenen Methoden ein großer Teil der im normalen Gewebe vorhandenen Harnsäure der Bestimmung entgehen. Im Gichtiker-Blut kann bald mehr oder weniger Harnsäure auch an Nuclein- oder Thyminsäure gebunden sein und entgeht auch der Analyse, der größte Teil jedoch ist nicht gebunden und wird ausgefällt. So entstehen die hohen Harnsäurewerte im Blute bei der Gicht.

Gegen die Minkowskische Annahme von einer so veränderten organischen Bindung von Harnsäure bei der Gicht muß schwer die Entdeckung Weintrauds ${ }^{3}$ ) ins Gewicht fallen; daß bei Thymusgenuß bei Gesunden gleichfalls hohe Harnsäurewerte im Blute mit den gebräuchlichen Methoden der

1) Goto, Diese Zeitschr., Bd. XXX, S. 473.

2) Minkowski, Verh. d. Kongresses f. innere Medizin, 1900.

$\left.{ }^{3}\right)$ Weintraud, Wiener klin. Rundschau, 1896, Bd. 1, S. 2, cit. Zentralbl. f. innere Medizin, 1896, S. 752. 
Harnsäurebestimmung zu finden sind. Wäre ein durchgehender prinzipieller Unterschied der Bindung der Harnsäure bei Gichtikern und Gesunden vorhanden, so müßte der Gesunde auch bei reichlicher Ausscheidung von Harnsäure in der Hauptsache unfällbare Harnsäure im Blut haben. Es bleibt noch der Ausweg quantitativer Unterschiede, der bis jetzt keinerlei Stütze in den Experimenten hat.

Alle Bemühungen, Anomalien der Harnsäureausscheidung im Harn in anfallsfreier Zeit und bei asthenischer Gicht zu finden, hatten bis jetzt keinen Erfolg. - Auf die Löslichkeitsund Mineralstoffwechseluntersuchungen im Harn gehen wir hier nicht weiter ein.

Man hat nun in neuerer Zeit, nach den Nucleinsäurearbeiten Kossels, durch die Untersuchungen Weintrauds, ${ }^{1}$ ) der zuerst die Steigerung der Harnsäureausscheidung im Harn nach Thymusgenuß feststellte, die Einwirkung reichlicher Thymusfütterung beim Gichtiker auf die Harnsäuremenge zum Gegenstand der Untersuchung gemacht.

Man ging von der ganz richtigen Voraussetzung aus, daß da am leichtesten ein Unterschied zu konstatieren sein wird, wo nach der Erfahrung am Gesunden die zahlenmäßig größten Mengen von Harnsäure gefunden werden. Wir zitieren von neuen Untersuchungen nach diesem Gesichtspunkte die Arbeiten von Vogt, ${ }^{2}$ ) $\operatorname{Reach}^{3}$ ) und Kaufmann und Mohr.4)

Vogt findet bei Zulage von $175 \mathrm{~g}$ Thymus pro Tag zur gemischten Nahrung beim Gichtiker eine um $106 \mathrm{mg}$ pro Tag geringere Harnsïureausscheidung als bei der gesunden Kontrollperson. in der Nachperiode aber pro Tag $179 \mathrm{mg}$ mehr bei der gesunden Kontrollperson, während die Vorperiode trotz merkwürdiger regelloser Verschiedenheit der Einzelwerte gleiche Durchschnittswerte für beide Versuchspersonen ergibt.

Reach fand bei Aufnahme von $150 \mathrm{~g}$ Kalbspankreas eine Vermehrung der Harnsäure im Harn von $119 \mathrm{mg}$. Er erwartet nach Burian und Schur angeblich $300 \mathrm{mg}$. Woher dieser Vergleichswert

1) Weintraud, Berl. klin. Wochenschrift 1895, S. 405.

2) Vogt, Arch. f. klin. Med. 1901, Bd. 71, S. 21.

3) Reach, Münchener med. Wochenschr. 1902, S. 1215. (Nach Zusammenstellung d. älteren Arbeiten.)

$\left.{ }^{4}\right)$ Kaufmann und Mohr, Arch. f. klin. Med. 1902, Bd. 74. 
kommt, ist mir nach Einsicht der Arbeiten von Burian und Schur nicht klar; es kann aber sein, daß mir die betreffende Stelle in den vorliegenden 300 Seiten Burian und Schur entgangen ist, sie wird von Reach nicht mit Angabe der Seitenzahl zitiert. Verfasser gibt nun selbst an, daß der Patient in der Aufnahme von Fleisch sehr unregelmäßig gewesen sei; dieses Faktum und der Mangel rechter, direkter Vergleichswerte veranlassen uns, die positiven Resultate etwas skeptisch in bezug auf ihre Zahlengrößen zu behandeln. Die Tatsache, daß die Ausscheidung auf $150 \mathrm{~g}$ Pankreas sehr gering war, scheint uns indessen auch so erwiesen.

Interessant ist bei Durchsicht der Zahlenreihen die Tatsache, daß in der Vorperiode ohne Pankreas $119 \mathrm{mg}$ Harnsäure im Durchschnitt weniger als in der Pankreasperiode ausgeschieden wurden, während in der pankreasfreien Nachperiode nur $18 \mathrm{mg}$, also ein Sechstel weniger Harnsäure als bei Pankreasnahrung ausgeschieden wird. Das heißt, die Pankreasnahrung wirkt 4 Tage nach, oder es kommen die oben erwähnten Fehlerquellen so stark zum Vorschein. Ein endgültiges Urteil über die quantitative Verschiedenheit ist ebensowenig aus Vogts Arbeit als aus der letzteren zu ziehen, wohl aber scheint aus beiden Untersuchungen hervorzugehen, daß bei Thymusgenuß der Gichtiker weniger Harnsäure ausgeschieden hat als der Gesunde, und diese Differenz nicht durch vermehrte oder verlängerte Ausscheidung in der Nachperiode ausglich. Interessant ist, daß in beiden Versuchen nach Pankreas- und Thymusfütterung ein Gichtanfall auftrat. Dieselben. Versuche wurden angestellt von Kaufmann und Mohr. ${ }^{1}$ ) Nur war in ihren Untersuchungen ein neuer Gesichtspunkt durch die physiologischen Arbeiten Hess' und Schmolls, ${ }^{2}$ ) Sivéns ${ }^{3}$ ) und Burians und Schurs ${ }^{4}$ ) getragen. Diese Autoren hatten endgültig festgestellt, daß der N-Gehalt der Nahrung für die ausgeschiedene Menge der Harnsäure irrelevant ist, daß allein die Höhe der Fleischzufuhr für die Höhe der Harnsäureausscheidung maßgebend ist.

Kaufmann und Mohr stellten nun neben anderen Untersuchungen, die uns hier nicht interessieren, Versuche an Gichtikern mit Thymusfütterung an. Leider steckten sie das Ziel, das sie $\mathrm{zu}$ erreichen wünschten, weiter, als es mir für ihre Methodik möglich erscheint, und so gelangten sie nicht zu dem gewünschten Resultat. Sie versuchten, ebenso wie Vogt, aus dem Verhalten des Harnsäure- und Phosphorsäurestoffwechsels etwas über «Retention» der Harnsäure zu erfahren. Sie empfinden die Schwierigkeiten ihrer Methode selbst, indem sie schreiben: «Trotzdem hegen wir, wie gesagt, ernstliche Zweifel, daß auf diesem Wege ein Ein-

1) Kaufmann und Mohr, Arch. f. klin. Med., Bd. 74 .

2) Hess und Schmoll, Arch. f. exp. Path., Bd. 37.

3) Sivén, Skandin. Arch. f. Physiol., Bd. 71, S. 122.

4) Burian und Schur, Pflügers Archiv, Bd. 80 und 87. 
blick in die Frage der Harnsäureretention bei Gichtkranken möglich ist.» - In ihrer Abhandlung, namentlich in dem Zahlenmaterial derselben, ist aber eine Fülle interessanter Tatsachen enthalten.

Auf Seite 589-594 finden wir die Resultate eines exakten Stoffwechselversuchs bei 5 Gichtikern zusammengestellt. Betrachten wir die Zahlen näher, so ergibt sich:

Tab. I. Ausscheidung der Harnsäure: 1. Bei fleischfreier Kost 4 Tage auffallend schwankend, am letzten Tag um $0,07 \mathrm{~g}$ ansteigend. Dann bei Thymuszulage $250 \mathrm{~g}$, am ersten Tag um $\mathbf{0 , 0 7 9} \mathrm{g}$ ansteigend, bei Burian ') um das 10 fache bei $310 \mathrm{~g}(0,78 \mathrm{~g}$ Anstieg), am zweiten um $\mathbf{0 , 3 3 8}$, am dritten erst um $\mathbf{0 , 6 1 5}$ ansteigend.

In der Nachperiode von 3 Tagen fallen die Werte sehr langsam, der Wert der Vorperiode wird überhaupt nicht wieder in 3 Tagen erreicht.

Tab. II. Bei Milch und Rahm 3 Tage Absinken, am letzten Tag kleine Steigung. Bei $300 \mathrm{~g}$ Thymuszulage: am ersten Tag Steigung um $\mathbf{0}, 29 \mathrm{~g} \overline{\mathrm{U}}$., am zweiten um 0,5 g $\overline{\mathrm{U}}$., am dritten um 0,52 g. In der Nachperiode fallen die Werte von 0,68 auf 0,439 und $0,562 \mathrm{~g} \overline{\mathrm{U}}$., auch hier wird der Minimalwert der kurzen Vorperiode nicht erreicht.

Im Versuch III wird bei Milchrahmdiät der Tageswert von $0,465 \mathrm{~g}$ erreicht, er erscheint am 4. Tag. Bei 300 g Thymus: Anstieg langsam, schwankende Zahlen; am 2. Tag höchster Wert. In der dritten Periode (Milch und Rahmdiät) am 1. Tag noch hoher Wert von 0,686 g Harnsäure, nach 3 Tagen Abfall auf Zahlen der Vorperiode. In der vierten Periode (Thymuszulage) Anstieg in den 3 Beobachtungstagen ganz minimal bei $300 \mathrm{~g}$ Thymuszulage auf $0,646,0,557$ ! und $0,764 \mathrm{~g}$ - das ist am 2. Tag nur Anstieg vom Minimum der Vorperiode von $\mathbf{0 , 1 2} \mathrm{g} \overline{\mathrm{U}}$. bei $300 \mathrm{~g}$ Thymus, während bei Burian am 2. Tag, wo also kumulierende Wirkung des Thymusgenusses erreicht wird, der Anstieg $0,98 \mathrm{~g}$ beträgt, also das Achtfache.

Ebenso im IV. Versuch sind die Differenzen zwischen Milchperiode und Thymusperiode auffallend klein, in der Nachperiode wird der Minimalwert der Vorperiode nicht erreicht. - Der V. Fall kann für unser Thema nicht direkt verwendet werden, auch er zeigt bei Fütterung mit reinem Nuclein am ersten Tag nach der Milchperiode nur eine Steigerung von $0,1 \mathrm{~g}$. In der Nachperiode sind die Werte höher als in der Nucleinperiode.

1) Burian und Schur, Pflügers Arch., Bd. 80, S. 312.

1) Anm.: Burian schied aus bei $310 \mathrm{~g}$ Thymus : 0,78 g Harnsäure mehr. Dann auf weitere $310 \mathrm{~g}$ : 0,98 g Harnsäure mehr. Der letztere Wert ist ein Mischwert vom 1. und 2. Fütterungstag, also zu hoch. Derselbe auf $300 \mathrm{~g}$ Schinken: 0,33 g Harnsäure mehr. Auf $300 \mathrm{~g}$ Rindfleisch: 0,27 g Harnsäure mehr. 
Dazu kommt noch die Beobachtung XLVI, S. 611. Wir haben hier in der Vorperiode ein Absinken auf 0,207 $\mathrm{g} \overline{\mathrm{U}}$., dann auf Zugabe von $300 \mathrm{~g}$ Thymus am ersten Tag Anstieg auf 0,681 $=0,47 \mathrm{~g} \mathrm{mehr,} \mathrm{am}$ zweiten auf $1,098 \mathrm{~g}$; die Werte der Nachperiode ohne Thymus sind 3-4 mal so hoch wie die der Vorperiode. Welche Vergleichswerte soll man da wählen? Die Nachperiode soll doch die Rückkehr zur Norm zeigen, sie ist nicht lang genug.

Verfasser hatten vorher S. $3 \check{4}$ ff. die sendogenen» Werte für $\overline{\mathrm{U}}$. bei Gicht bestimmt, auffallend erscheint ihnen die «Inkonstanz der Alloxurkörperwerte». Ich habe bei den Harnsäurewerten keine Inkon$\operatorname{stanz}$ konstatieren können, sie zeigen zumeist abfallende Tendenz. Die Normalbestimmung des «ektogenen» Anteils auf Fleisch- und Fischfütterung (S. 157-159) zeigt eine für normale, von uns nie gefundene Inkonstanz der Werte für Harnsäureausscheidung; ich kann mir das nicht anders erklären, als daß Fleisch verschiedener Tiere von Tag zu Tag verfüttert wurde, es ist vielleicht auch die Differenz auf die Verwendung von Rekonvaleszenten zu diesen Versuchen zu schieben.

Schwankungen bei konstanter fleischfreier Diät, wie sie in der Tabelle S. 158 enthalten sind, haben wir bei gesunden Versuchspersonen nie beobachtet.

Wir entnehmen zusammenfassend den Versuchen von Kaufmann und Mohr: Bei Thymusgenuß erscheint beim Gichtiker ein langsamerer und stets geringerer Anstieg der Harnsäure im Harn als bei den gesunden Personen, die Burian und Schur untersuchten. Der Abfall vollzieht sich wie bei dem oben besprochenen Fall von Reach in so langsamer Weise, daß in 3 tägiger Nachperiode die Werte der Vorperiode garnicht wieder erreicht werden. Die mangelnde Länge der Versuche ist für ihre Verwertung sehr störend, der Zweck der Nachperiode ist doch grade, die Werte der Vorperiode wieder zu erreichen.

Wir wissen bis jetzt von dem Verhalten der Harnsäure bei der Gicht:

1. Die Harnsäure lagert sich in den Gelenken ab.

2. Die Harnsäuremenge im Harn steigt bei gemischter Nahrung in und gleich nach dem Anfall an.

3. Die Harnsäuremenge im Harn ist nach Genuß von Thymus und Pankreas kleiner als bei gesunden Kontrollpersonen. 
Ist damit für die Lehre von der Harnsäureausscheidung bei der Gicht, um den hier zu behandelnden Punkt herauszugreifen, das Erreichbare erreicht?

Es schien uns notwendig, die Beobachtung der Harnsäureausscheidung am Krankenbett der Gicht noch einmal unter genauer Beobachtung der Diät zu studieren, um so doch vielleicht Abweichungen von der Norm feststellen zu können.

Als Vergleichsmaterial mit Gesunden hätte uns nur das kleine Material von Burian und Schur zur Verfügung gestanden. Es genügte für unsere Zwecke nicht und Herr cand. med. Pfeil übernahm es, die Ausscheidung der Harnsäure am Gesunden zu studieren. Die Ergebnisse seiner Untersuchungen sind niedergelegt in der vorstehenden Arbeit: «Über den Einfluß der Nahrungsaufnahme auf die Ausscheidung der Harnsäure.»

Wir können aus den Pfeilschen Untersuchungen entnehmen:

1. Bei fleischfreier Nahrung stellt sich der Gesunde in 1-2 Tagen auf das Minimum seiner Harnsäureausscheidung ein.

2. Die Form seiner 3stündlichen Harnsäureausscheidungskurve gleicht einer geraden Linie mit einer mehr oder weniger starken Steigung am Morgen (siehe Kurve A u. B getönt).

3. Bei fleischhaltiger Kost steigt nach der Fleischaufnahme die Tagesmenge der ausgeschiedenen Harnsäure sofort auf ziemlich hohe Werte an. Bei einem Versuchsquantum von $340-350 \mathrm{~g}$ Fleisch betrug die Steigerung pro Tag $400-500 \mathrm{mg}$ Harnsäure (siehe Kurve A und B weiß).

4. Die Reaktion trat auch nach fleischfreier längerer Periode sofort am 1. Tage ein.

5. Die Form der Ausscheidungskurve war auch bei Fleischnahrung eine charakteristische, individuell unabhängige.

Die Kurven A und B, aus den Pfeilschen Untersuchungen entnommen, sind zum Vergleich neben die in dieser Untersuchung gefundenen Gichtkurven gestellt. Sämtliche Kurven sind genau im gleichen Maßstabe ausgeführt, also direkt zu vergleichen. 
Wir beabsichtigen, die Ausscheidungsweise der Harnsäure bei der Gicht genau nach derselben Methode zu studieren, wie es Pfeil bei Gesunden getan hat.

Die früheren Forscher hatten in bezug auf die Ausscheidungstageswerte in anfallsfreier Zeit keine charakteristischen Abweichungen von der Norm konstatieren können.

Muß deshalb die Ausscheidung ebenso verlaufen, wie bei Gesunden?

Wir können eine gleiche Ausscheidungstagesmenge auf sehr verschiedene Weise erhalten. Der Organismus kann sich einer Menge A in 24 Stunden durch stetes Tröpfeln entledigen, kann aber dieselbe Menge auch ausscheiden durch entsprechend kürzeren Ablauf im kräftigen Strahl. Von diesem Ausscheidungsmodus verrät uns die Beschäftigung mit der Tagesmenge nichts. Und doch kann gerade der Ausscheidungsmodus uns den Weg weisen zur Feststellung des pathologischen Verhaltens der Harnsäure, ganz abgesehen davon, daß es nach den Untersuchungen von Soetbeer und Ibrahim ${ }^{1}$ ) möglich sein kann, daß auch stundenweise Verzögerung schon von intensivster Wirkung ist.

Diese Überlegung führte uns auf die Methode der Untersuchung in Stundenwerten.

Aus den Untersuchungen der physiologischen Stundenwerte sahen wir, daß beim Übergang von fleischreicher zu fleischfreier Kost eine stetig abfallende Kurve sich zeigte, die 2 Tage abfiel, ehe sie den für fleischfreie Kost charakteristischen Minimalwert erreichte. Wir ersehen daraus, daß nach fleischreicher Periode noch längere Zeit größere Mengen von Harnsäure zur Ausscheidung kommen.

Die natürliche Folgerung war, daß wir uns durch gleiche Anordnung der Aufeinanderfolge von fleischreicher und fleischfreier Kost zu überzeugen suchten, ob diese Zeit anhaltender Mehrausscheidung beim Gichtiker gleichermaßen und in derselben Form stattfand.

1) Soetbeer und Ibrahim, Über das Schicksal eingeführter Harnsäure im menschlichen Organismus. Diese Zeitschr., Bd. XXXV, S. 1. 
Wir sahen ferner, wie überall in der klinischen Beschreibung bei dem plötzlichen Wechsel von fleischfreier und fleischreicher Kost, auf die üble Wirkung von Milchkuren und anderen strengen, einseitigen Diätkuren hingewiesen wurde. Diese Beobachtungen, die wir auch selbst Gelegenheit hatten zu machen, veranlaßten uns, besonderes Augenmerk zu legen auf die dem Diätwechsel direkt folgenden Perioden.

Nimmt man eine Stauung an, betrachtet man den Gichtiker als einen mit Harnsäure gefüllten Eimer (um das praktische alte Bild noch einmal zu gebrauchen), so konnte die Überlaufsmenge pro Tag bei gleichmäßiger Lebensweise, und die ist ja die Vorbedingung unserer Stoffwechselversuche, ganz gleichmäßig sein. Ganz anders aber mußte sich die U-Quantität verhalten, wenn es gelang, den Eimer zu entleeren und die nächsten Tage nach der Entleerung, die Tage bis zum Überlaufen, zu untersuchen, sie mußten uns charakteristische Ausfälle zeigen.

Auf Grund solcher Überlegungen ist die Methodik für unsere Beobachtungen über die Ausscheidung der Harnsäure bei der Gicht aufgestellt.

\section{Methodik.}

Wir haben die dreistündigen Werte der Harnsäureausscheidung bei Gicht bestimmt:

1. Bei fleischfreier Kost.

2. Bei gemischter Kost mit reichlich Fleisch.

3. Wir haben in 3-Stundenkurven den Übergang studiert von fleischfreier zu fleischhaltiger Kost und umgekehrt.

Sämtliche Analysen sind am frischen Harn innerhalb 24 Stunden nach der Methode Salkowski-Ludwig gemacht. Benutzte Analysenmenge und verbrauchte Titrationssäure ist in den Tabellen angegeben, um dem Leser Material für eigene Kritik über die Bewertung der Differenzen zu geben.

Die Harnsäure wurde nicht gewogen, sondern in den chlorfrei gewaschenen Krystallen der Stickstoffgehalt bestimmt und die Harnsäure berechnet.

Die Patienten beobachteten teils Bettruhe, teils durften sie sich nach Belieben in Haus und Garten bewegen.

Das Material war nur mit größter Mühe zu beschaffen, die Untersuchungen sind unter den schwierigsten äußeren Verhältnissen ange- 
stellt. Ich möchte gleich hier meinem Freunde und Kollegen Kasbaum in Heidelberg für die mir auf das freundschaftlichste zur Verfügung gestellten Fälle seiner Privatpraxis meinen herzlichsten Dank aussprechen.

Ebenso danke ich ergebenst Herrn Prof. Lenhartz für den mir zur Verfügung gestellten Fall P. im Eppendorfer Krankenhaus und für die mir gütigst erteilte Erlaubnis, in dem Laboratorium des Krankenhauses meine Analysen $\mathrm{zu}$ machen. Ich danke Herrn Prof. Lenhartz ferner für das Interesse, das er meinen Untersuchungen entgegengebracht hat, sowie den Herren Dr. Schumm und Wagner für die Hilfe im Laboratorium und die Unterstützung bei der Einrichtung und Überwachung des Versuchs auf der Station.

Es bleibt mir noch übrig, Herrn Prof. Krehl in Tübingen für das Material, das er mir zur Verfügung stellte, sowie für die Mittel seines Laboratoriums herzlichst zu danken. Meinem früheren Chef Herrn Prof. Vi erordt in Heidelberg danke ich ergebenst für die Erlaubnis, nach meinem Fortgang aus der Klinik noch das Laboratorium für die Untersuchung des Falles B. und F. benutzen zu dürfen. Schließlich danke ich ergebenst der hohen medizinischen Fakultät zu Heidelberg für den mir zur Ausführung dieser Untersuchungen bereitwilligst gewährten Urlaub von einem Semester.

Es ist bei aller Gichtforschung die Beschaffung des Krankenmaterials eine der größten Schwierigkeiten. Gicht trifft man meistens nur bei Privatpatienten. In diesen Verhältnissen liegt es auch begründet, daß es oft notwendig ist, die Untersuchungsperioden kürzer zu gestalten als es im Interesse der Sache wünschenswert ist. Wir glauben, durch sachkundige Auswahl der Untersuchungstage mit unseren Perioden die ausschlaggebenden, charakteristischen Zeiten getroffen zu haben.

Alles Weitere und etwaige Abweichungen oder Verluste sind erwähnt bei der Anführung der Versuchsresultate.

\section{Versuch I.} anfall :

Bernhard C., 76 Jahre alt, hatte am 2. April 1903 einen Gicht-

Arteriosklerose, Emphysem. - Patient hat viel gereist, lebte immer sehr gut, vor ca. 20 Jahren "Gicht» (nach Aussage des Arztes) in den Fingern, Verdickung der Gelenke noch zu sehen, kein Knirschen, etwas behinderte Funktion. Später Schmerzen im Hüftgelenk.

Mai 19028 Tage lang Schmerzen im linken Knie. Ein englischer Arzt konstatierte "Rauhigkeit im Kniegelenk. Mitte Februar 1903 Schmerzen im linken Knie, Behandlung mit Ichthyol-Vaseline. Am 1. April Champagner, etwas reichlicher als gewöhnlich, abends war 
das Knie empfindlich, in der Nacht heiß und starke Schmerzen. Dabei fieberfrei und ohne Appetit. Am linken Knie Gelenkspalte stark druckempfindlich, ebenso Tibiakopf ganz umschrieben. Keine Rötung, Funktion ganz beschränkt. Passive Bewegungen sehr schmerzhaft. In den nächsten Tagen noch starke Schmerzhaftigkeit, Besserung in ca. 14 Tagen. Während der Behandlung Schmerzen in der rechten Schulter nach einem warmen Bade.

Harn bei wiederholter früherer und späterer Untersuchung eiweißund zuckerfrei.

Patient hatte in den Vortagen wenig Appetit und hatte leichte Suppen, etwas Ei, Fleisch, Kompott, Tee, Weißbrot gegessen.

Am 3. April, mittags $1^{30}$, begann er nach folgender Vorschrift $\mathrm{zu}$ essen :

\section{Fleischfreie Periode:}

$7^{30}$. 1/2 Liter Milch, 2 Brötchen, $30 \mathrm{~g}$ Butter, 1 Zigarre. $1^{30} .150 \mathrm{~g}$ Kartoffeln (roh gewogen), $25 \mathrm{~g}$ Butter, 3 Eier; Pudding: $50 \mathrm{~g}$ Reis, $50 \mathrm{~g}$ Butter, $30 \mathrm{~g}$ Zucker, $1 \mathrm{Ei}$; $20 \mathrm{~g}$ Brot, $50 \mathrm{~g}$ Salat, Himbeersaft. $-7^{30} .50 \mathrm{~g}$ Käse, $30 \mathrm{~g}$ Butter, 2 Brötchen, 1/2 Liter Milch.

Der Urin wurde aufgefangen und analysiert um 6, 9 und 12 Uhr vormittags und um $3,6,9$ und 12 Uhr nachmittags. Die Nahrung wurde beibehalten am 4. und 5. April.

Am Montag den 6. April nahm Patient

Fleischhaltige Diät:

$8^{80}$. $1^{1 / 2}$ Brötchen, $25 \mathrm{~g}$ Butter, $25 \mathrm{~g}$ Zucker, $400 \mathrm{ccm}$ Kaffee. 130. $220 \mathrm{~g}$ Fleisch, $15 \mathrm{~g}$ Butter, $60 \mathrm{~g}$ Kartoffeln, 1 Apfelsine. $4^{30}$. $400 \mathrm{ccm}$ Kaffee, $25 \mathrm{~g}$ Zucker, $1 \frac{1}{2}$ Brötchen, $30 \mathrm{~g}$ Butter. $8^{30}$. $30 \mathrm{~g}$ Brot, $100 \mathrm{~g}$ Schinken, $10 \mathrm{~g}$ Käse, $400 \mathrm{~g}$ Tee, $25 \mathrm{~g}$ Zucker, $20 \mathrm{~g}$ Butter.

Wir haben also einen 76 Jahre alten Patienten, der jahrelang gänzlich beschwerdefrei ist, plötzlich einen Gichtanfall nach langer anfallsfreier Periode bekommt, während des Anfalls auf seine Harnsäureausscheidung untersucht. Fieber bestand nicht. Urin: eiweiß- und zuckerfrei.

Patient schied aus : 
Einfluß der Nahrungsaufnahme auf die Ausischeidung der Harnsäure. 39

Tabelle I. Kurve I.

\begin{tabular}{c|c|c|c|c|c}
\hline Zeit & Harnmenge & $\begin{array}{c}\text { Analysen- } \\
\text { menge }\end{array}$ & $\begin{array}{c}1_{10} \text { norm. } \\
\text { Säure }\end{array}$ & $\begin{array}{c}\text { Harnsäure } \\
\text { in g }\end{array}$ & $\begin{array}{c}\text { Harnsäure } \\
\text { pro Std. in g }\end{array}$ \\
\hline
\end{tabular}

\begin{tabular}{|c|c|c|c|c|c|c|c|}
\hline \multicolumn{8}{|c|}{ 4. April. (Fleischfrei.) } \\
\hline 3 & Uhr & nachm. & 95 & 80 & 9,6 & 0,053 & 0,018 \\
\hline 6 & $»$ & 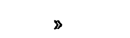 & 117 & 100 & $10, \check{5}$ & 0,057 & 0,019 \\
\hline 9 & $\triangleright$ & $\triangleright$ & 110 & 90 & 9,8 & 0,055 & 0,018 \\
\hline 12 & $\gg$ & mittern. & 55 & 45 & 5,9 & 0,033 & 0,011 \\
\hline 6 & $\triangleright$ & vorm. & 93 & 80 & 10,0 & 0,054 & 0,009 \\
\hline
\end{tabular}

5. April. (Fleischfrei.)

\begin{tabular}{|c|c|c|c|c|c|c|c|}
\hline 9 & Uhr & vorm. & 78 & 60 & 8,5 & 0,051 & 0,017 \\
\hline 12 & $»$ & mittags & 133 & 100 & 7,6 & 0,047 & 0,016 \\
\hline 3 & $»$ & nachm. & 43 & 35 & 3,9 & 0,022 & 0,007 \\
\hline 6 & $»$ & 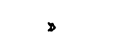 & 114 & 100 & 10,2 & 0,054 & 0,018 \\
\hline 9 & $»$ & , & 109 & 90 & 9,4 & 0,053 & 0,018 \\
\hline 12 & $\gg$ & mittern. & 132 & 100 & 8,7 & 0,053 & 0,018 \\
\hline 6 & $»$ & vorm. & 191 & 100 & 8,0 & 0,071 & 0,012 \\
\hline
\end{tabular}

6. April. (Fleischtag.)

\begin{tabular}{|c|c|c|c|c|c|}
\hline 9 Uhr vorm. & 216 & 100 & 5,2 & 0,052 & 0,017 \\
\hline $12 》$ & 85 & 70 & 5,6 & 0,031 & 0,010 \\
\hline 3 u. 6 U. nachm. & & & erlo & & \\
\hline 9 Uhr nachm. & 57 & 45 & 5,1 & 0,030 & 0,010 \\
\hline $12 \gg$ mittern. & $15 \check{~}$ & 100 & 1,6 & 0,011 & 0,004 \\
\hline $6 \gg$ vorm. & 210 & 100 & 7,9 & 0,077 & 0,013 \\
\hline
\end{tabular}

7. April. (Fleisch.)

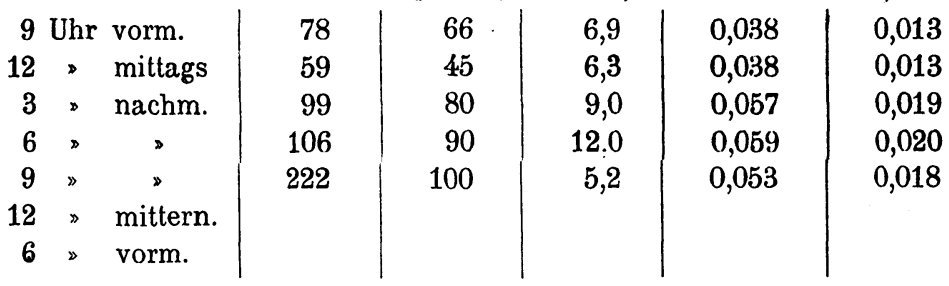

Wir entnehmen aus den Zahlen der Tabelle I die Werte einer lückenlosen 24 stündigen Periode ohne Fleisch vom 5. zum 6. April und eine ebensolche entsprechende lückenlose Periode mit Fleisch vom 6. zum 7. April. Beide von $9 \mathrm{Uhr}$ nachm. bis 9 Uhr nachm.

Der Patient schied ohne Fleisch in 24 Stunden 0,316 g Harnsäure aus, bei einer Aufnahme von 320 g Fleisch dagegen 
nur eine Tagesmenge von $0,310 \mathrm{~g}$. Die Differenz von $0,006 \mathrm{~g}$ Harnsäure fällt in die Fehlergrenzen der Methode.

Der Patient hat also bei einer Aufnahme von 320 g Fleisch die gleiche Menge Harnsäure wie ohne Fleischnahrung ausgeschieden.

Wenn wir die Formen der getönten 3stündlichen Ausscheidungskurve I bei fleischfreier Kost und der weißen Kurve bei Fleischnahrung betrachten, so sehen wir, daß die charakteristischen Anstiege, die wir in der vorstehenden Abhandlung von Pfeil konstatieren konnten, nicht eingetreten sind.

Wir sehen vielmehr, wie sowohl die Harnsäureportion 9 Uhr nachm. am 6. April nach $220 \mathrm{~g}$ Fleisch als auch die Portion 12 Uhr nachm. nach Neuaufnahme von 100 g Schinken um $8^{30}$ mit die niedrigsten sind, die überhaupt ausgeschieden wurden. Ebenso fehlt eine Steigerung am 7. April. Die Form der Kurve von 12 Uhr mittags bis 9 Uhr abends, die die charakteristische Reaktion auf die Fleischaufnahme enthalten müßte, zeigt genau dieselbe Form und Höhe, wie die fleischfreie Kurve vom 4. April zu derselben Zeit.

Die Aufnahme von Wasser war reichlich und unbeschränkt, die Diurese zeigte keinen Zusammenhang mit dem Schwanken der Kurve. Sie war z. B. um 12 Uhr mitternachts, wo die ganz geringe Menge ausgeschieden wurde, sehr reichlich, $155 \mathrm{ccm}$ in 3 Stunden. Ganz klein war sie jedoch bei der geringen Menge am 5. April, wo sie nur $43 \mathrm{ccm}$ Harn betrug. Es wäre ja möglich, daß zu geringe Diurese die Harnsäuremengenausscheidung herabdrückt, jedenfalls aber sieht man, daß reichliche Diurese die Ausscheidung nicht im günstigen Sinne beeinflussen muß.

\section{Versuch II (Kurve II, Tabelle II).}

Der zweite Versuch betraf eine 62 Jahre alte Dame, die Arteriosklerose hat, ohne Veränderung des Harns. - 1897, 1. Anfall: Linkes Metatarso-phalangeal-Gelenk. - Nach 4 Monaten weiterer Anfall an gleicher Stelle, die sich in regelmäßigen Intervallen wiederholten, alle 4 Monate. 1899: Gehäuftere Anfälle, immer im selben Gelenk. 1900: Sicher (Dr. Kasbaum) beobachteter Gichtanfall. 1902: ohne Anfälle, 
Einfluß der Nahrungsaufnahme auf die Ausscheidung der Harnsäure.

Empfindlichkeit in dem betreffenden Gelenk nie ganz geschwunden. Sehr vorsichtige, mäßige, regelmäßige Lebensweise.

Der Versuch erstreckte sich vom 26. April bis 1. Mai.

Vom 26. April bis 28. April incl. genoß Patientin fleischfreie Diät, die Aufnahme von Wasser und Mineralwasser war ihr und auch allen anderen Patienten nach Bedarf völlig freigestellt. Die Diät war quantitativ genau dieselbe wie in Fall I; wir unterlassen es, sie hier noch einmal zu wiederholen. Ebenso war die Diät mit Fleisch dieselbe wie in Fall $\mathrm{I}$.

Während des ganzen Versuches fühlte sich die Patientin vollkommen wohl.

Die ausgeschiedene Harnsäuremenge haben wir zusammengestellt auf folgender Tabelle II.

\begin{tabular}{l|l|c|c|c|c}
\hline \hline Zeit & Harnmenge & $\begin{array}{c}\text { Analysen- } \\
\text { menge }\end{array}$ & $\begin{array}{c}{ }_{1 / 10} \text { norm. } \\
\text { Säure }\end{array}$ & $\begin{array}{c}\text { Harnsäure } \\
\text { in } g\end{array}$ & $\begin{array}{c}\text { Harnsäure } \\
\text { pro Std. in } g\end{array}$ \\
\hline
\end{tabular}

\begin{tabular}{|c|c|c|c|c|c|c|}
\hline \multicolumn{7}{|c|}{ 26. April. (Fleischfrei.) } \\
\hline 12 & Uhr mittags & 93 & 93 & 7,0 & 0,032 & 0,011 \\
\hline 3 & "nachm. & 110 & 100 & 10,3 & 0,052 & 0,017 \\
\hline 6 & $》$ & 93 & 93 & 9,9 & 0,046 & 0,015 \\
\hline 9 & » & 80 & 75 & 5,7 & 0,028 & 0,009 \\
\hline 12 & » mittern. & 158 & 100 & $8, \check{5}$ & 0,062 & 0,02 \\
\hline 6 & » vorm. & 122 & 100 & 9,6 & 0,054 & 0,009 \\
\hline & Pro Tag & $6 \check{6} 6$ & 一 & - & 0,274 & - \\
\hline
\end{tabular}

27. April. (Fleischfrei.)

\begin{tabular}{|c|c|c|c|c|c|c|}
\hline 9 & Uhr vorm. & 75 & 75 & 6,6 & 0,030 & 0,010 \\
\hline 12 & » mittags & 126 & 126 & 8,1 & 0,037 & 0,012 \\
\hline 3 & nachm. & 112 & 112 & 9,7 & 0,045 & 0,015 \\
\hline 6 & $\gg$ & $8 \tilde{~}$ & 85 & 8,7 & 0,040 & 0,013 \\
\hline 9 & » & 97 & 97 & 8,8 & 0,041 & 0,013 \\
\hline 12 & » mittern. & 102 & 102 & 8,5 & 0,039 & 0,013 \\
\hline 6 & » vorm. & 240 & 100 & 8,1 & 0,09 & 0,015 \\
\hline & Pro Tag & 837 & - & - & 0,322 & - \\
\hline
\end{tabular}

28. Apriz. (Fleischfrei.)

\begin{tabular}{|c|c|c|c|c|c|c|}
\hline 9 & hr vorm. & 60 & 60 & 5,2 & 0,024 & 0,008 \\
\hline 2 & » mittags & 128 & 128 & 5,8 & 0,027 & 0,009 \\
\hline 3 & » nachm. & 93 & 93 & 5,7 & 0,026 & 0,009 \\
\hline 6 & $\triangleright$ & 142 & 142 & 117 & 0,054 & 0,018 \\
\hline 9 & $\triangleright$ & 124 & 124 & 5,8 & 0,027 & 0,009 \\
\hline 2 & » mittern. & 142 & 142 & 6,8 & 0,031 & 0,010 \\
\hline 6 & vorm. & 168 & 168 & 11,0 & 0,051 & 0,008 \\
\hline & Pro Tag & 857 & - & - & 0,240 & - \\
\hline
\end{tabular}


Franz Soetbeer,

\begin{tabular}{c|c|c|c|c|c} 
Zeit & Harnmenge & $\begin{array}{c}\text { Analysen- } \\
\text { menge }\end{array}$ & $\begin{array}{c}{ }_{1}{ }_{10} \text { norm. } \\
\text { Säure }\end{array}$ & $\begin{array}{c}\text { Harnsäure } \\
\text { in g }\end{array}$ & $\begin{array}{c}\text { Harnsäure } \\
\text { proStd. in g }\end{array}$ \\
\hline
\end{tabular}

\begin{tabular}{|c|c|c|c|c|c|c|c|}
\hline \multicolumn{8}{|c|}{ 29. April. (Fleis ch.) } \\
\hline 9 & $\mathrm{Jhr}$ & vorm. & 65 & 65 & 7,0 & 0,032 & 0,011 \\
\hline 12 & > & mittags & 130 & 130 & 12,9 & 0,06 & 0,020 \\
\hline 3 & $\triangleright$ & nachm. & 90 & 90 & 11,7 & 0,054 & 0,018 \\
\hline 6 & $\gg$ & » & 156 & 100 & 15,4 & 0,111 & 0,037 \\
\hline 9 & $»$ & ه & 150 & 100 & 11,9 & 0,082 & 0,028 \\
\hline 12 & & mittern. & 150 & 100 & 11,7 & 0,081 & 0,027 \\
\hline 6 & & vorm. & 200 & 100 & 12,4 & 0,11 อ̆ & 0,019 \\
\hline & & Pro Tag & 941 & - & - & 0,535 & - \\
\hline
\end{tabular}

\begin{tabular}{rll|r}
9 & Uhr vorm. & 100 \\
12 & $\searrow$ & mittags & 130 \\
3 & nachm. & 110 \\
6 & $\searrow$ & 194 \\
9 & $\searrow$ & & 140 \\
12 & mittern. & 176 \\
6 & vorm. & 260 \\
& & Pro Tag & 1110
\end{tabular}

30. April. (Fleisch.)

\begin{tabular}{|c|c|c|c|c|c|c|}
\hline \multicolumn{7}{|c|}{ 1. Mai. (Fleisch.) } \\
\hline 9 & Uhr vorm. & 100 & 100 & 12,5 & 0,058 & 0,019 \\
\hline 2 & mittags & 140 & 140 & 16,2 & 0,075 & 0,025 \\
\hline 3 & \ nachn. & 144 & 144 & 23,0 & 0,110 & 0,035 \\
\hline 6 & $\triangleright$ & 94 & 94 & 14,4 & 0,066 & 0,022 \\
\hline 9 & $\diamond$ & 106 & 106 & 16,5 & 0,076 & 0,025 \\
\hline 2 & - mittern. & 204 & 100 & 10,3 & 0,097 & 0,032 \\
\hline 6 & vorm. & 258 & 100 & 9,7 & 0,116 & 0,019 \\
\hline & Pro Tag & 1046 & - & - & 0,598 & - \\
\hline
\end{tabular}

Die Tagessumme der Fleischtage erhebt sichin diesem Versuche um 0,295 am ersten Tag, um 0,387 am zeiten Tag, um 0,358 am dritten Tag für $300 \mathrm{~g}$ Fleisch und \& Stunden über die Mitte der Tagesmenge der fleischfreien Tge. Diese Steigerung der Tagesmenge entspricht völlig der Norm Ähnliche Zahlen haben Pfeil und auch Burian und Schu in ihren oben zitierten Versuchen erhalten.

Sehen wir uns die 3 stündige Menge an, dieForm der Tagesausscheidungskurve, so finden wir, daß die fleischfreie Kurve am ersten Tage ähnlich wie in Fall 2 der 'feilschen Untersuchungen nach der Abendmahlzeit, obwohl sie gnz fleischfrei ist, einen Anstieg zeigt. Am nächsten Morge fehlt die 
in der Norm beobachtete Morgensteigerung vollständig, noch auffälliger am nächsten Tage. Die Steigerungen nach den Mahlzeiten bleiben aus, am 27. beschreibt die Tageskurve eine fast gerade Linie, die am 28. durch eine einzige plötzliche Steigerung wieder nach einer Mahlzeit ohne Fleisch unterbrochen wird. Die Steigerung trat so unmotiviert ein, daß wir uns durch Wiederholung der Analysen von ihrer Richtigkeit glaubten überzeugen zu müssen.

Wir haben also hier während der fleischfreien Zeit eine Kurve, die der Norm erspricht mit zwei unmotivierten Steigerungen von zwei 3 stündlichen Mengen in $3 \times 24$ Stunden. Der Grund dieser vereinzelten Beobachtungen ist natürlich nicht einmal vermutungsweise anzugeben.

Auffallend bleibt, daß ebenso wie in Fall I die Morgensteigerung der Norm völlig fehlt.

Vom 29. April bis 1. Mai wurden pro Tag $300 \mathrm{~g}$ Fleisch in 2 Portionen um $1^{30}$ und $7^{30}$ gegeben.

Wir finden am 29. ebenso wie am 30. eine prompte Steigerung auf die Aufnahme. Die Kurven verlaufen völlig normal. Auffallend ist an ihnen jedoch, daß auf den Kaffee am 29. April eine deutliche Steigerung eintritt, die in der Norm und auch bei den andern Gichtfällen ausbleibt. Abweichend ist ferner der sehr hohe Nachtwert 2-6 am 30. April. Jedoch ist trotz dieser kleineren Abweichungen in der Höhe der Typus der normalen Kurve erhalten.

Anders ist es am dritten Tage, am 1. Mai. Hier kommt die Ausscheidung, wenn man so sagen darf, aus dem Takte. Die Portion um 3 Uhr nachmittags, die bei Fleischaufnahme um $1^{30}$ die niedrigste nach den Normalbeobachtungen sein müßte, ist die höchste des Tages, und die Portion um 6 Uhr nachmittags, die die höchste sein müßte, bleibt ganz niedrig, ebenso bleibt die Steigerung noch in den nächsten drei Stunden bis $9 \mathrm{Uhr}$ aus und endlich in der Portion von 9 Uhr abends bis Mitternacht haben wir einen nur mäßigen Anstieg.

Vergleichen wir die Kurve des 1. Mai mit einer der Normalfleischkurven, so sehen wir auf den'ersten Blick, daß auch sie stark von der Norm abweicht. 
Wir haben hier also einen chronischen Gichtfall leichtester Form mit lange ausbleibenden Anfällen und Beschwerden in anfalls- und beschwerdefreier Zeit untersucht und konnten konstatieren, daß Unregelmäßigkeiten in der Stundenausscheidung der Harnsäure vorkommen, wie wir sie in der Norm nicht beobachten konnten.

Im dritten Falle handelte es sich um einen Patienten, bei dem die Ätiologie des Bleis, er war 3 Jahrzehnte Schriftsetzer, nicht ganz von der Hand zu weisen ist. Auch hier handelt es sich um einen Gichtfall, der ebenso wie Fall II äußerst milde verlief, vor 2 Jahren hatte die Krankheit zum letztenmal Beschwerden verursacht.

Wir lassen das Notwendige aus der Krankengeschichte hier folgen:

Fall C.

Patient 58 Jahre alt, Schriftsetzer, Tübingen.

.. Seit 4 Jahren keine Berufsgeschäfte mehr. Keine Bleikolik, keine Lähmungen. Vor 30 Jahren Behandlung von Liebermeister wegen Neurasthenie, Krampfanfälle im Magen. Schiebt seine neurasthenischen Beschwerden auf Masturbation vom 19.-28. Jahr, dann Heirat, immer sehr nervös.

Erster Anfall vor $4^{1 / 2}$ Jahren, über Nacht starke Schmerzen in der großen Zehe, Rötung und Schwellung, \& Tage krank. Der zweite Anfall 2 Jahre später in derselben Zehe, aber 8 Tage dauernd. Kur in Wildbad 1901. Seitdem nie mehr Beschwerden, die auf Gicht hinweisen. Tophus am Ohr.

Urin eiweiß- und zuckerfrei, ohne Formbestandteile.

Versuch vom 22.-26. Juni.

Der Patient erhielt als Nahrung vom 22.-25. Juni: $7^{30}$. 1/2 Liter Milch, 2 Milchbrote, $1 \mathrm{Ei}, 30 \mathrm{~g}$ Butter. $-1^{80} .100 \mathrm{~g}$ geröstete Kartoffeln, $50 \mathrm{~g}$ Salat, 3 Spiegeleier; Reispudding: $50 \mathrm{~g}$ Reis, 2 Eier, $30 \mathrm{~g}$ Butter, $1 / 4$ Liter Milch, $20 \mathrm{~g}$ Zucker, Himbeersaft. $-7^{50}$. 1/2 Liter Milch, 2 Milchbrote, 2 Eier, $20 \mathrm{~g}$ Käse, $30 \mathrm{~g}$ Butter. - Am 26. Juni: $7^{30} .400 \mathrm{ccm}$ Tee, $30 \mathrm{~g}$ Butter, 2 Brötchen, 1 Ei. $-1^{30}$. $250 \mathrm{~g}$ Fleisch, $30 \mathrm{~g}$ Butter, $100 \mathrm{~g}$ Kartoffeln, $50 \mathrm{~g}$ Salat, 1 Brötchen. $-7^{30} .2$ Brötchen, $30 \mathrm{~g}$ Butter, $30 \mathrm{~g}$ Käse, $100 \mathrm{~g}$ Schinken, $400 \mathrm{ccm}$ Tee.

Wir haben den Versuch insofern vereinfacht, daß wir die ersten 3 Tage der fleischfreien Diät nicht analysiert haben und erst den 4. Tag, wo wir annehmen konnten, daß der Minimalwert der Harnsäureausscheidung erreicht sei, haben wir als Versuchstag dreistündlich analysiert.

\section{Die Harnanalysen ergaben:}


Einfluß der Nahrungsaufnahme auf die Ausscheidung der Harnsäure. 45

Tabelle III. Kurve III.

\begin{tabular}{c|c|c|c|c|c}
\hline Zeit & Harnmenge & $\begin{array}{c}\text { Analysen- } \\
\text { menge }\end{array}$ & $\begin{array}{c}{ }^{1} / 5 \text { norm. } \\
\text { Säure }\end{array}$ & $\begin{array}{c}\text { Harnsäure } \\
\text { in } \mathbf{g}\end{array}$ & $\begin{array}{c}\text { Harnsäure } \\
\text { pro Std. in g }\end{array}$ \\
\hline
\end{tabular}

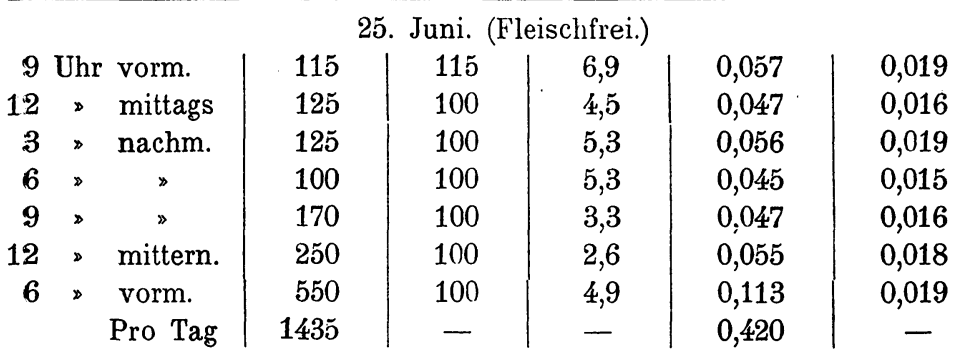

26. Juni. (Fleisch.)

\begin{tabular}{|c|c|c|c|c|c|c|c|}
\hline $\begin{array}{r}9 \\
12\end{array}$ & Uhr & $\begin{array}{l}\text { vorm. } \\
\text { mittags }\end{array}$ & $\left.0,240^{1}\right)$ & \multicolumn{2}{|c|}{$\begin{array}{l}\text { Verlust } \\
\text { Verlust }\end{array}$} & 0,104 & - \\
\hline 3 & , & nachm. & 170 & 100 & 3,7 & 0,053 & 0,018 \\
\hline 6 & $»$ & > & 200 & 100 & 4,5 & 0,076 & 0,025 \\
\hline 9 & $»$ & D & 260 & 100 & 3,1 & 0,068 & 0,023 \\
\hline 12 & $\gg$ & mittern. & 225 & 100 & 3,8 & 0,075 & 0,025 \\
\hline 6 & $»$ & vorm. & 655 & 100 & 2,9 & 0,160 & 0,027 \\
\hline & & Pro Tag & 1750 & - & - & 0,536 & - \\
\hline
\end{tabular}

Man ersieht aus Tabelle und Kurve III, daß der fleischfreie Tag den Typus der Normalkurve zeigt, die gerade Linie, aber wiederum ohne die Steigerung am Morgen. Im allgemeinen liegt die Kurve der Ausscheidung von Harnsäure bei fleischfreier Nahrung bei diesem Patienten ziemlich hoch. Die Tagesmenge beläuft sich auf $0,420 \mathrm{~g}$ Harnsäure. Wir ersehen aus dieser Zahl sehr leicht, wie mit mittleren Tagesharnsäurewerten bei der Gicht garnichts anzufangen ist. Durch die bei jedem, sei es Gesunden oder Kranken, gänzlich anders liegende Minimalkurve wird natürlich jede direkte Vergleichung zu einer Unmöglichkeit.

Es ist nur möglich, nach Feststellung der individuellen Minimalkurve die Ausscheidung der Harnsäure zu beurteilen nach der Form und Menge ihrer Steigerung nach Fleischaufnahme.

Sehr zu bedauern ist es, daß der etwas hysterische Patient aus irgend welchen Gründen den Verlust von den 2 Morgen-

1) Ergänzt aus den Zahlen des 25. Juni. 
portionen am nächsten Tage verursachte. Wir glauben aber für diese 2 Portionen, ohne einen Fehler zu machen, die 2 Morgenportionen des fleischfreien Tages einsetzen zu können, da es uns doch nur auf die Demonstration der Wirkung der Fleischaufnahme ankommt, die erst um $6 \mathrm{Uhr}$ nachmittags eintreten kann.

Wir sehen hier wiederum, wie nach der Aufnahme von Fleisch, sowohl in der Quantität (die Steigerung beträgt in 24 Stunden 0,116 g) wie auch in der Form, eine Abweichung von der Norm stattfindet. Wir vermissen den typischen starken Anstieg nach der Fleischaufnahme. Wir sehen, wie in einem Falle, der seit 2 Jahren keine gichtischen Beschwerden mehr hat, dennoch eine Abweichung von der gewöhnlichen Reaktion der Harnsäureausscheidung auf Fleischaufnahme statthat.

Die Wertschätzung dieser Versuche fassen wir am Ende der Abhandlung zusammen, sie ist je nach dem Grade der Abweichung für das, was wir zu zeigen bestrebt sind, natürlich eine sehr verschiedene. Wir haben aber absichtlich alle untersuchten Fälle hier zusammengestellt. Es kam uns keineswegs darauf an, eine völlige Übereinstimmung in dem Verhalten aller Gichtiker nachzuweisen, das widerspräche dem klinischen Charakter der wechselndsten aller Erkrankungen, sondern wir wollten unsere klinischen Beobachtungen an den uns zu Gebote stehenden Gichtfällen der verschiedensten Intensität hier zusammenstellen.

Im Falle IV handelt es sich um einen Amerikaner, der seit dem Jahre 1893 von typischen akuten Anfällen geplagt wird und vor ca. 6 Wochen den letzten leichten Anfall gehabt hatte. Im Moment, wo er zur Beobachtung kam, war er beschwerdefrei.

Wir konnten also hier erwarten, etwas zu erfahren über die Zeit, die bis jetzt jeder Beobachtung getrotzt hatte. Jeder Versuch, irgend eine Anomalie der Ausscheidung des Urins in anfalls- und beschwerdefreier Zeit $\mathrm{zu}$ finden, muß bis jetzt als gescheitert betrachtet werden.

Die Anamnese des Patienten ergab folgende Tatsachen:

Fall 0. (Med. Klinik zu Tübingen).

Der erste Anfall im Jahre 1893 im rechten Knöchelgelenk, typisch in der Nacht aufgetreten; die Entzündung des Gelenkes dauerte 1 Woche. Nach 2-3 Tagen Anfall im Knie derselben Seite. Im Jahre 1894 wieder 
ein Anfall, ganz plötzlich, von gleicher Stärke in denselben Gelenken; er dauerte 8-10 Tage. Patient wurde nach Karlsbad geschickt; in Karlsbad in der dritten Woche heftiger Anfall im linken Knöchel.

Dann bis 1896 frei. Von 1896 ab jedes Jahr ein Anfall, und zwar immer im Januar und immer in denselben Gelenken. Im Jahre 1900 zum erstenmal auch wieder im linken Knöchel. Im Jahre 1901 wurde Patient nach Neuenahr geschickt, am Ende der Kur (im Juli) sehr heftiger Anfall am linken Knöchel, er war jedoch in 3 Tagen völlig abgeklungen. Das Fieber war bei allen Anfällen mäßig hoch.

1902 frei von Anfällen.

1903 erster Anfall, leicht im linken Fuß und Knie, dann im April (Paris) sehr leichter Anfall im linken Knöchel. Patient brauchte seine Beschäftigung nicht zu unterbrechen.

Dann 14 Tage später noch ein ganz leichter Anfall im linken Knie. Patient suchte dann im Juni Prof. Krehl auf.

Im Jahre 1897 soll ein kleiner Stein mit dem Harn abgegangen sein.

In Urin in den ersten 2 Tagen vor dem Versuch Spuren von Eiweiß, die auf Bettruhe verschwinden. Keine Formbestandteile. Kein Zucker.

\section{Versuch vom 19.-24. Juni.}

Der Patient erhielt als Nahrung am 19.-22. Juni: $7^{30}$. 1/2 Liter Milch, 2 Milchbrote, $1 \mathrm{Ei}, 30 \mathrm{~g}$ Butter. - $1^{30} .100 \mathrm{~g}$ geröstete Kartoffeln, $50 \mathrm{~g}$ Salat, 3 Spiegeleier; Reispudding: $50 \mathrm{~g}$ Reis, 2 Eier, $30 \mathrm{~g}$ Butter, 1/4 Liter Milch, $20 \mathrm{~g}$ Zucker, Himbeersaft. $-7^{30}$. 1/2 Liter Milch, 2 Milchbrote, 2 Eier, $20 \mathrm{~g}$ Käse, $30 \mathrm{~g}$ Butter. - Vom 23.-24. Juni : $7^{30}$. $400 \mathrm{ccm}$ Tee, $30 \mathrm{~g}$ Butter, 2 Milchbrote, 1 Ei. - 1*0. $250 \mathrm{~g}$ Fleisch (roh gewogen und gebraten), $30 \mathrm{~g}$ Butter, $100 \mathrm{~g}$ Kartoffeln, $50 \mathrm{~g}$ Salat, 1 Brötchen. $-7^{30}$. 2 Brötchen, $30 \mathrm{~g}$ Butter, $30 \mathrm{~g}$ Käse, $100 \mathrm{~g}$ Schinken, $400 \mathrm{ccm}$ Tee.

Die Analysenergebnisse der 3 stündlichen Harnmengen sind in folgender Tabelle und Kurve zusammengefaßt:

Tabelle IV. Kurve IV.

\begin{tabular}{c|c|c|c|c|c}
\hline \hline Zeit & Harnmenge & $\begin{array}{c}\text { Analysen- } \\
\text { menge }\end{array}$ & $\begin{array}{c}1_{5} \text { norm. } \\
\text { Säure }\end{array}$ & $\begin{array}{c}\text { Harnsäure } \\
\text { in g }\end{array}$ & $\begin{array}{c}\text { Harnsäure } \\
\text { pro Std. in g }\end{array}$ \\
\hline
\end{tabular}

\begin{tabular}{|c|c|c|c|c|c|c|}
\hline & \multicolumn{6}{|c|}{ 20. Juni. (Fleischfrei.) } \\
\hline 9 & Uhr vorm. & 124 & 100 & 8,9 & 0,090 & 0,030 \\
\hline 12 & » mittags & 140 & 100 & 6,3 & 0,074 & 0,025 \\
\hline 3 & » nachm. & 120 & 100 & 8,7 & 0,088 & 0,029 \\
\hline 6 & $》$ & 135 & 100 & 8,3 & 0,094 & 0,031 \\
\hline 9 & , & 100 & 100 & 6,9 & 0,058 & 0,019 \\
\hline 12 & - mittern. & 110 & 110 & 7,6 & 0,064 & 0,021 \\
\hline 6 & $\bowtie$ vorm. & 160 & 100 & 6,5 & 0,087 & 0,015 \\
\hline & Pro Tag & 889 & - & - & 0,565 & - \\
\hline
\end{tabular}




\begin{tabular}{l|l|c|c|c|c}
\hline Zeit & Harnmenge & $\begin{array}{c}\text { Analysen- } \\
\text { menge }\end{array}$ & $\begin{array}{c}1 / 5 \text { norm. } \\
\text { Säure }\end{array}$ & $\begin{array}{c}\text { Harnsäure } \\
\text { in g }\end{array}$ & $\begin{array}{c}\text { Harnsäure } \\
\text { pro Std. in g }\end{array}$ \\
\hline
\end{tabular}

21. Juni. (Fleischfrei.)

\begin{tabular}{rcc|c|c|c|c|c}
9 & Uhr & vorm. & 210 & 100 & 5,8 & 0,102 & 0,034 \\
12 & $\searrow$ & mittags & 150 & 100 & 7,9 & 0,100 & 0,033 \\
3 & $\searrow$ & nachm. & 185 & 100 & 4,3 & 0,067 & 0,022 \\
6 & $\searrow$ & $\searrow$ & 175 & 100 & 4,0 & 0,059 & 0,019 \\
9 & $\searrow$ & $\searrow$ & 125 & 100 & 4,2 & 0,044 & 0,015 \\
12 & $\searrow$ & mittern. & 305 & 100 & 1,7 & 0,022 & 0,007 \\
6 & vorm. & 300 & 100 & 3,4 & 0,086 & 0,014 \\
& & Pro Tag & 1450 & - & - & 0,480 & -
\end{tabular}

22. Juni. (Fleischfrei.)

\begin{tabular}{rcc|c|c|c|c|c}
9 & Uhr vorm. & 320 & 100 & 2,6 & 0,070 & 0,023 \\
12 & $\gg$ & mittags & 125 & 100 & 6,9 & 0,072 & 0,024 \\
3 & $\gg$ & nachm. & 240 & 100 & 3,5 & 0,071 & 0,023 \\
6 & $\gg$ & $\triangleright$ & 160 & 100 & 4,3 & 0,058 & 0,019 \\
9 & $\gg$ & $\gg$ & 100 & 100 & 4,4 & 0,037 & 0,012 \\
12 & mittern. & 340 & 100 & 1,8 & 0,051 & 0,017 \\
6 & $>$ & vorm. & 460 & 100 & 4,3 & 0,083 & 0,014 \\
& & Pro Tag & 1745 & - & - & 0,442 & -
\end{tabular}

23. Juni. (Fleisch.)

\begin{tabular}{|c|c|c|c|c|c|c|c|}
\hline 9 & Uhr & vorm. & 270 & 100 & 2,9 & 0,066 & 0,022 \\
\hline 12 & $»$ & mittags & 140 & 100 & 5,5 & 0,065 & 0,022 \\
\hline 3 & » & nachm. & 120 & 100 & 3,3 & 0,053 & 0,018 \\
\hline 6 & $»$ & $»$ & 125 & 18 & 0,9 & 0,053 & 0,018 \\
\hline 9 & $\triangleright$ & $》$ & 150 & 100 & 3,8 & 0,048 & 0,016 \\
\hline 12 & $»$ & mittern. & 130 & 100 & 4,7 & 0,051 & 0,017 \\
\hline 6 & $\triangleright$ & vorm. & 440 & 100 & 4,0 & 0,148 & 0,024 \\
\hline & & Pro Tag & 1445 & - & - & 0,484 & - \\
\hline
\end{tabular}

24. Juni. (Fleisch.)

\begin{tabular}{rcl|c|c|c|c|c}
9 & Uhr & vorm. & 225 & 100 & 3,9 & 0,074 & 0,024 \\
12 & $\searrow$ & mittags & 110 & 110 & 9,2 & 0,077 & 0,026 \\
3 & $\searrow$ & nachm. & 180 & 100 & 5,7 & 0,086 & 0,029 \\
6 & $\gg$ & $\searrow$ & 170 & 100 & 6,0 & 0,086 & 0,029 \\
9 & $\searrow$ & $\gg$ & 150 & 100 & 4,4 & 0,055 & 0,018 \\
12 & $\searrow$ & mittern. & 310 & 100 & 3,4 & 0,089 & 0,030 \\
6 & $\searrow$ & vorm. & 360 & 100 & 3,9 & 0,118 & 0,020 \\
& & Pro Tag & 1505 & - & - & 0,585 & -
\end{tabular}

Am 19. Juni ließen wir den Kranken bei Bettruhe fleischfrei essen und analysierten den Harn noch nicht. Er wurde 
jedoch am 20., wo er eiweißfrei war, dreistündlich aufgefangen und analysiert.

In den getönten Kurven sehen wir deutlich den langsamen Abstieg der Harnsäurewerte bei fleischfreier Nahrung. Der erste Kurventag, der 20. Juni, ist der zweite fleischfreie Tag. Wir können den Abstieg durch die Tagesmengen aus Tabelle IV illustrieren. Am 20. Juni haben wir $0,565 \mathrm{~g}$, am 21. Juni 0,480 , am 22. Juni 0,442 . Es ist möglich, daß bei längerer Fortsetzung fleischfreier Ernährung wir noch einen etwas niedrigeren Wert erhalten hätten, viel aber wäre sicher die Harnsäureausscheidung nicht gesunken.

Was die Form der Kurven anbelangt, so zeigen sie wiederum die starken Anstiege am Morgen, wie bei der normalen Ausscheidung im Gegensatz zu den vorher beobachteten Kranken und dem noch folgenden Falle. Wir haben hier eine von Tag zu Tag sich kontinuierlich verringernde Tagesmenge von ausgeschiedener Harnsäure. Keinesfalls aber verringert sich auch die Stundenmenge successive, wir haben auch hier wieder Berge und Täler der Kurve, wir haben Anstiege am nächsten Morgen weit über Werte, die am Tage vorher erreicht waren. Wir weisen auf dieses Verhalten der Kurven hin, um zu zeigen, wie kompliziert die Verhältnisse liegen.

Der Körper entledigt sich eines Stoffes, aber er kann sich seiner nicht kontinuierlich entledigen, die Ausschwemmungsmöglichkeit der Harnsäure ist von Faktoren abhängig, von denen wir noch keine Ahnung haben.

Am vierten Tag ist die gerade Linie, die wir vom Normalversuch kennen und in den vorstehenden Versuchen wiedergefunden haben, noch nicht erreicht. Wir mußten jedoch aus leicht ersichtlichen Gründen mit der Fleischnahrung beginnen, da sonst eventuell an der Ungeduld des Patienten der ganze Versuch gescheitert wäre.

Was ergab nun die Fleischfütterung? Hätten wir die Kurve am 23. Juni getönt, so konnte man meinen, die fleischfreie Kost wäre noch einen Tag weiter verabreicht, um das Minimum der Ausscheidung und die Form der geraden Linie $\mathrm{zu}$ erreichen.

Hoppe-Seyler's Zeitschrift f. physiol. Chemie. XL. 
Dem ist aber nicht so, die so gerade verlaufende, einen Harnsäureausscheidungswert von 0,484 g repräsentierende Kurve entspricht einer Fleischaufnahme von $320 \mathrm{~g}$.

Wir haben mit dem tiefsten Werte der fleischfreien Kurve nur eine Differenz von $0,042 \mathrm{~g}$ Harnsäure, während die beiden anderen fleischfreien Tage höhere oder gleiche Werte zeigen.

Der nächste Fleischtag bringt die Erhöhung auf $0,585 \mathrm{~g}$, um 0,02 $\mathrm{g}$ höher als der erste fleischfreie Tag, um $0,143 \mathrm{~g}$ höher als der niedrigste fleischfreie Tag. Welche Differenz aber gegenüber den Normalkurven zeigen diese beiden Tage und wie verwischt, wie gänzlich untypisch, regellos verläuft die Ausscheidung der Harnsäure, wenn wir die Kurve mit den beigedruckten Normalkurven A und B vergleichen.

Wir haben hier einen Fall beobachtet, der klinisch die Zeichen einer subakuten Gicht zeigte, die Beobachtungszeit ist völlig anfallstrei und von dem letzten Anfall sechs Wochen entfernt.

Unsere Beobachtung der Harnsäureausscheidung ergab bei fleischfreier Kost ein etwas langsameres als bei Gesunden Absinken der 24 stündigen Werte für die Harnsäureausscheidung bei gleicher Form der dreistündlichen Ausscheidungskurve wie im Normalfall B. Die gerade Linie wurde nicht erreicht.

Dagegen zeigte sich sowohl in der Tagesmenge, wie in der Stundenausscheidungsform ein fundamentaler Unterschied mit den Beobachtungen am Normalen. Auch in anfallsfreier Zeit reagierte dieser Gichtiker 24 Stunden fast garnicht auf Zufuhr von $320 \mathrm{~g}$ Fleisch und in den zweiten 24 Stunden also auf die doppelte Portion von $640 \mathrm{~g}$ Fleisch sehr schwach, ungefähr um ein Sechstel wie beim Normalen. Die beim Normalen stets auftretende starke Reaktion der Stundenkurve auf Fleischzufuhr blieb natürlicherweise auch völlig aus. Die erste Kurve zeigt die reaktionslose Form der fleischfreien Kurve, die zweite eine völlig regellose uncharakteristische Form. Wir konstatieren also auch in anfallsfreier Zeit bei diesem Gichtiker ein Abweichen von der Norm bei der Ausscheidung der Harnsäure.

Der fünfte Kranke repräsentierte eine Gicht mit schwersten Gelenkerscheinungen ohne Erkrankung der Niere. 
Ich entnehme der sehr ausführlichen Kranlkengeschichte (Dr. Wa g n er) das für die Charakterisierung des Falles Notwendige.

Patient P. ist Malergehilfe, 41 Jahre alt. 1876 als Malerlehrling hatte er Bleikolik, 8 Wochen bettlägerig, später keine Erscheinungen mehr trotz Fortsetzung des Berufs.

1882. 1. Gichtanfall, Schmerzen in beiden Fersen, Schwellung. Daver 3-4 Tage.

Alle Vierteljahr Wiederholung, an Intensität nachlassend. Seit einigen Jahren «Knoten» in den Ohren. Steife Fingergelenke, Schmerzen in Handgelenken und Füßen.

Der Status enthält eine genaue Beschreibung der weitverbreiteten Lokalisation der Erkrankung und eine Beschreibung der Funktionsbehinderung der Gelenke und ihrer Dislokationen. Der Urin ist frei von Eiweiß und Zucker.

Bei einmaliger Fleischmahlzeit am Tag und sonstiger fleischfreier Kost nach dem Versuch fühlt Patient sich leidlich wohl und wird nach ca. zwei Wochen gebessert entlassen.

Die Beobachtung erstreckte sich vom 15.-22. Mai, zur Zeit eines akuten Gichtanfalls, der ohne Fieber verlief. Der Urin war immer eiweißund zuckerfrei.

Der Patient wurde genau in derselben Weise ernährt, wie die beiden vorhergehenden Fälle. Er aß vom 15.--18. Mai inclusive fleischfrei und erhielt an den folgenden Tagen um $1^{\text {Bo }}$. Uhr $250 \mathrm{~g}$ Beefsteak und um $7^{30} 100 \mathrm{~g}$ Schinken zugelegt.

Drei Stundenwerte der Harnsäureausscheidung und ihre Analysenbelege sind ebenso wie in den vorhergehenden Fällen in nachstehender Tabelle und Kurve $\mathrm{V}$ zusammengefaßt.

\begin{tabular}{c|c|c|c|c|c}
\hline Zeit & Harnmenge & $\begin{array}{c}\text { Analysen- } \\
\text { menge }\end{array}$ & $\begin{array}{c}1_{\text {/o norm. }} \text { Săure } \\
\text { Să }\end{array}$ & $\begin{array}{c}\text { Harnsäure } \\
\text { in g }\end{array}$ & $\begin{array}{c}\text { Harnsäure } \\
\text { pro Std. in g }\end{array}$ \\
\hline
\end{tabular}

(16. Mai $0,25 \mathrm{~g} \overline{\mathrm{U}}$. - 17. Mai $0,24 \mathrm{~g} \overline{\mathrm{U}}$.)

18. Mai. (Fleischfrei.)

\begin{tabular}{|c|c|c|c|c|c|c|}
\hline 9 & Uhr vorm. & 172 & 100 & 5,3 & 0,038 & 0,013 \\
\hline 12 & » mittags & 154 & 100 & 5,4 & 0,035 & 0,012 \\
\hline 3 & nachm. & 186 & 100 & $4, \check{0}$ & 0,035 & 0,012 \\
\hline 6 & $\triangleright$ & 208 & 100 & 5,1 & 0,045 & 0,015 \\
\hline 9 & 》 & 188 & 100 & 4,9 & 0,040 & 0,013 \\
\hline 10 & $\triangleright$ & 80 & 80 & 3,8 & 0,016 & 0,016 \\
\hline 6 & » vorm. & 380 & 100 & 4,2 & 0,067 & 0,011 \\
\hline & Pro Tag & 1368 & - & - & 0,276 & - \\
\hline
\end{tabular}




\begin{tabular}{c|c|c|c|c|c}
\hline \hline Zeit & Harnmenge & $\begin{array}{c}\text { Analysen- } \\
\text { menge }\end{array}$ & $\begin{array}{c}\left.{ }^{1}\right|_{10} \text { norm. } \\
\text { Säure }\end{array}$ & $\begin{array}{c}\text { Harnsäure } \\
\text { in } g\end{array}$ & $\begin{array}{c}\text { Harnsäure } \\
\text { pro Std. in g }\end{array}$ \\
\hline
\end{tabular}

\begin{tabular}{|c|c|c|c|c|c|c|c|}
\hline \multicolumn{8}{|c|}{ 19. Mai. (Fleischfrei.) } \\
\hline 9 & Uhr & vorm. & 215 & 100 & 4,3 & 0,039 & 0,013 \\
\hline 12 & 》 & mittags & 215 & 100 & 5,0 & 0,045 & 0,015 \\
\hline 3 & 》 & nachm. & 205 & 100 & 3,6 & 0,031 & 0,010 \\
\hline 6 & 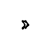 & $\gg$ & 315 & 100 & 5,2 & 0,069 & 0,023 \\
\hline 9 & $\triangleright$ & $\triangleright$ & 205 & 100 & 5,2 & 0,047 & 0,016 \\
\hline 1.2 & , & mittern. & 195 & 100 & 4,4 & 0,036 & 0,012 \\
\hline \multirow[t]{2}{*}{6} & , & vorm. & 365 & 100 & 4,0 & 0,061 & 0,010 \\
\hline & & Pro Tag & 1.715 & - & - & 0,328 & - \\
\hline
\end{tabular}

19. Mai. (Fleischfrei.)

20. Mai. (Fleisch.)

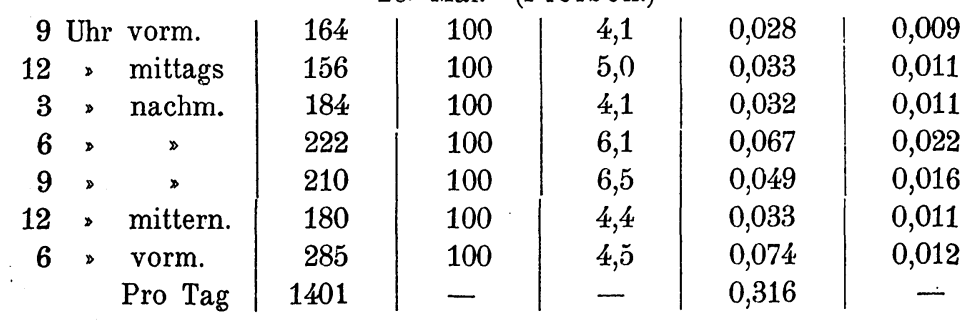

21. Mai. (Fleisch.)

\begin{tabular}{|c|c|c|c|c|c|c|}
\hline 9 & Uhr vorm. & 165 & 100 & 4,6 & 0,032 & 0,011 \\
\hline 12 & $\gg$ mittags & 260 & 100 & 5,8 & 0,063 & 0,021 \\
\hline 3 & , nachm. & 150 & 100 & 2,7 & 0,017 & 0,006 \\
\hline 6 & $\triangleright$ & 206 & 100 & 5,7 & 0,049 & 0,016 \\
\hline 9 & , & 210 & 100 & 5,4 & 0,049 & 0,016 \\
\hline 12 & , mittern. & 174 & 100 & 4,1 & 0,030 & 0,010 \\
\hline 3 & vorm. & 320 & 100 & 2,2 & $0,0: 30$ & 0,005 \\
\hline & Pro Tag & 1485 & - & - & 0,270 & - \\
\hline
\end{tabular}

22. Mai. (Fleisch.)

\begin{tabular}{|c|c|c|c|c|c|c|}
\hline 9 & Uhr vorm. & 150 & 100 & 4,7 & 0,030 & 0,010 \\
\hline 12 & $\gg$ mittags & 160 & 100 & 4,5 & 0,030 & 0,010 \\
\hline 3 & n nachm. & 150 & - & 一 & 0,017 & - \\
\hline 6 & \& & 210 & 100 & 3,9 & 0,034 & 0,011 \\
\hline 9 & 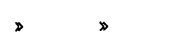 & 220 & 100 & 6,6 & 0,061 & 0,020 \\
\hline 12 & $\bowtie$ mittern. & 170 & 100 & 3,9 & 0,028 & 0,009 \\
\hline 6 & v vorm. & 300 & 100 & 4,4 & $0,0 \tilde{5}$ & 0,009 \\
\hline & Pro Tag & 1360 & - & - & 0,255 & - \\
\hline
\end{tabular}

Der Kranke hatte sich schon vor der Beobachtungszeit fast fleischfrei genährt und so zeigten die Gesamtwerte der Harnsäureausscheidung am 16. und 17. Mai keine abfallende Tendenz, sie betrugen 0,25 und 0,24 g Harnsäure pro Tag. 
Die in Einzelwerten am 18. Mai bestimmte 24stündige Menge betrug 0,276 g. Die kleine Steigerung haben wir stets beobachtet bei den Bestimmungen in Einzelwerten, sie muß ihren Grund im Analysieren haben, ist aber so klein, daß sie auf die Bewertung der Resultate keinen Einfluß ausübt.

Die fleischfreie Kurve zeigt bei ziemlich niedrigem Tageswerte die uns bekannte Form der fleischfreien Kurve ohne die Steigerung am Morgen.

Am 19. Mai erscheint auch hier auf Aufnahme von Kaffee eine Steigerung der Harnsäureausscheidung, auch 250 g Fleisch bewirken einen Anstieg, der aber im Vergleich zum normalen minimal ist, die $100 \mathrm{~g}$ Schinken am Abend lassen die Kurve völlig unbeeinflußt, sie bleibt niedrig.

Verfolgen wir nun die Ausscheidung der nächsten drei Tage bei gleicher Aufnahme, so sehen wir, wie die Dreistundenausscheidung regellos schwankt, bald tritt auf Fleischaufnahme eine kleine Steigerung an der richtigen Stelle ein (20. Mai), bald bleibt sie völlig aus (21. und 22. Mai). Der Genuß der $100 \mathrm{~g}$ Schinken am Abend ist stets ohne irgend welchen Einfluß auf die Ausscheidung der Harnsäure. Die Kurven haben keine charakteristische Form, sie bleiben überhaupt abnorm niedrig und schwanken regellos bald auf bald ab. - Das ganze Niveau der Kurven liegt da, wo wir es sonst nur bei fleischfreier Nahrung $\mathrm{zu}$ finden gewohnt sind.

Auf Aufnahme von $350 \mathrm{~g}$ Fleisch pro Tag beträgt die Mehrausscheidung an Harnsäure von 24 Stunden

$$
\begin{array}{ccr}
\text { am } & \text { 19. Mai } & 0,052 \\
» & 20 . & \\
» & 21 . & 0,040 \\
» & 22 . & -0,006 \\
& 2 & -0,021
\end{array}
$$

Das heißt: Bei Aufnahme von $350 \mathrm{~g}$ Fleisch scheidet der beobachtete Kranke im Anfall an den beiden ersten Tagen 52 und $40 \mathrm{mg}$ Harnsäure mehr aus als an fleischfreien Tagen, und an den folgenden Tagen, wo man eine kumulierende Wirkung noch erwarten konnte, scheidet der Kranke 6 und $21 \mathrm{mg}$ weniger Harnsäure in 24 Stunden aus als bei fleischfreier Kost.

Diese Zahlen fallen in die Fehlergrenzen. Wir können mit Sicherheit entnehmen, daß die Aufnahme von $350 \mathrm{~g}$ 
Fleisch bei dem Gichtkranken im Anfall ohne Einwirkung auf die Harnsäureausscheidung war.

\section{Zusammenfassung.}

Der Einfluß der Nahrungsaufnahme bei arthritis urica auf die Ausscheidungstages- und Stundenwerte der Harnsäure ist ein von der Norm abweichender.

Bei chronischer Gicht, ohne Gelenkbeschwerden und ohne Veränderung der Nieren, sehen wir bei fleischfreier Kost kleinere Abweichungen von der normalen Form der 3stelligen Ausscheidungskurve, die in plötzlichen Steigerungen der 3 Stundenwerte sich zeigen.

Bei Aufnahme von $300 \mathrm{~g}$ Fleisch zur gemischten Nahrung sehen wir bald Störungen der Regelmäßigkeit der Ausscheidung, bald regellose und quantitativ stark verminderte Ausscheidung.

Bei akuter Gicht, im Anfall ohne Fieber und ohne Nierensymptome, beobachteten wir bei fleischfreier Kost geringe Abweichungen von der Norm. Die Kurven näherten sich mehr oder weniger der Form der geraden Linie. Die an den Normalkurven beobachtete Morgensteigerung der 3stündigen Kurve fehlte meistens bei den Gichtkranken.

Bei Aufnahme von 300-350 g Fleisch konnten wir im akuten Anfall die deutlichsten Störungen der Ausscheidung der Harnsäure feststellen.

Nicht nur blieb die quantitative Erhöhung der Harnsäure im Harn ganz oder fast ganz aus, die Kurven zeigten auch in ihrer Form eine völlige Regellosigkeit, die auf tiefgehende Störungen der Ausscheidung der Harnsäure hinweist.

Den Versuchen fehlt die Breite. Der Einzelversuch hätte über viel längere Perioden ausgedehnt werden müssen. Die Zahl der Versuche hätte bei einer in ihrer Erscheinungsform so wechselnden Erkrankung eine viel größere sein müssen.

Die Fehler wurden durch die äußeren Umstände bedingt. Jedenfalls verspricht der eingeschlagene Weg der Untersuchung die Möglichkeit, das klinische Bild der Gicht in bezug auf die Harnsäureausscheidung richtig zu stellen und zu vertiefen. 
Erklärung der Kurven.

$5 \mathrm{~mm}$ der Abcisse $=1$ Stunde.

$1 \mathrm{~mm}$ der Ordinate $=1 \mathrm{mg}$ Harnsäure.

Die Kurven sind von links nach rechts zu lesen. Jede Reihe umfaßt 24 Stunden.

Anfang links morgens 8 resp. 6 Uhr.

Ende rechts morgens 8 resp. 6 Uhr. 
Brought to you by | Purdue University L Authenticated

Download Date | 5/26/15 2:20 Al 


\section{KurveA .}

$\downarrow$

Milch

leit: :
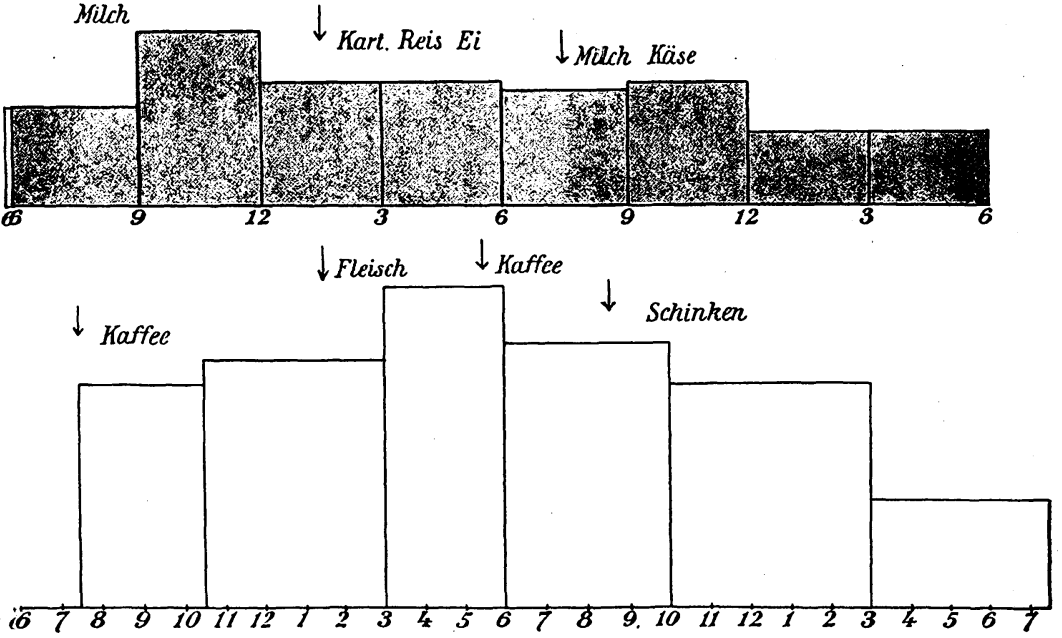

\section{Kurve $B$.}

$\downarrow$ Milch
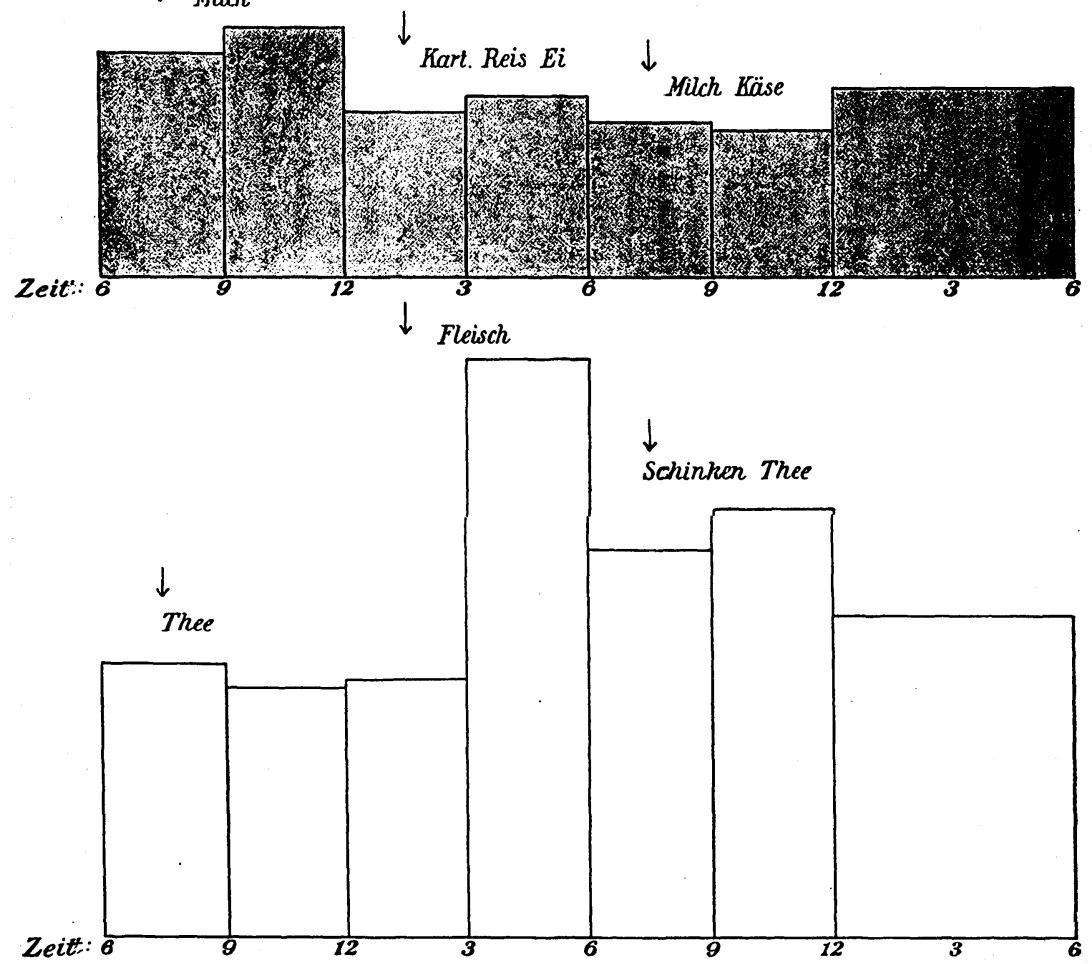

Hloppe-Seyler's Zeitschrift für physiologische Chemie. Band XL, Tafel 7.

Zu «Soetbeer, Über den Einfluß der Nahrungsaufnahme auf die Ausscheidung der Harnsäure bei Arthritis urica». 
Brought to you by | Purdue University $L$ Authenticated

Download Date | 5/26/15 2:20 Al 


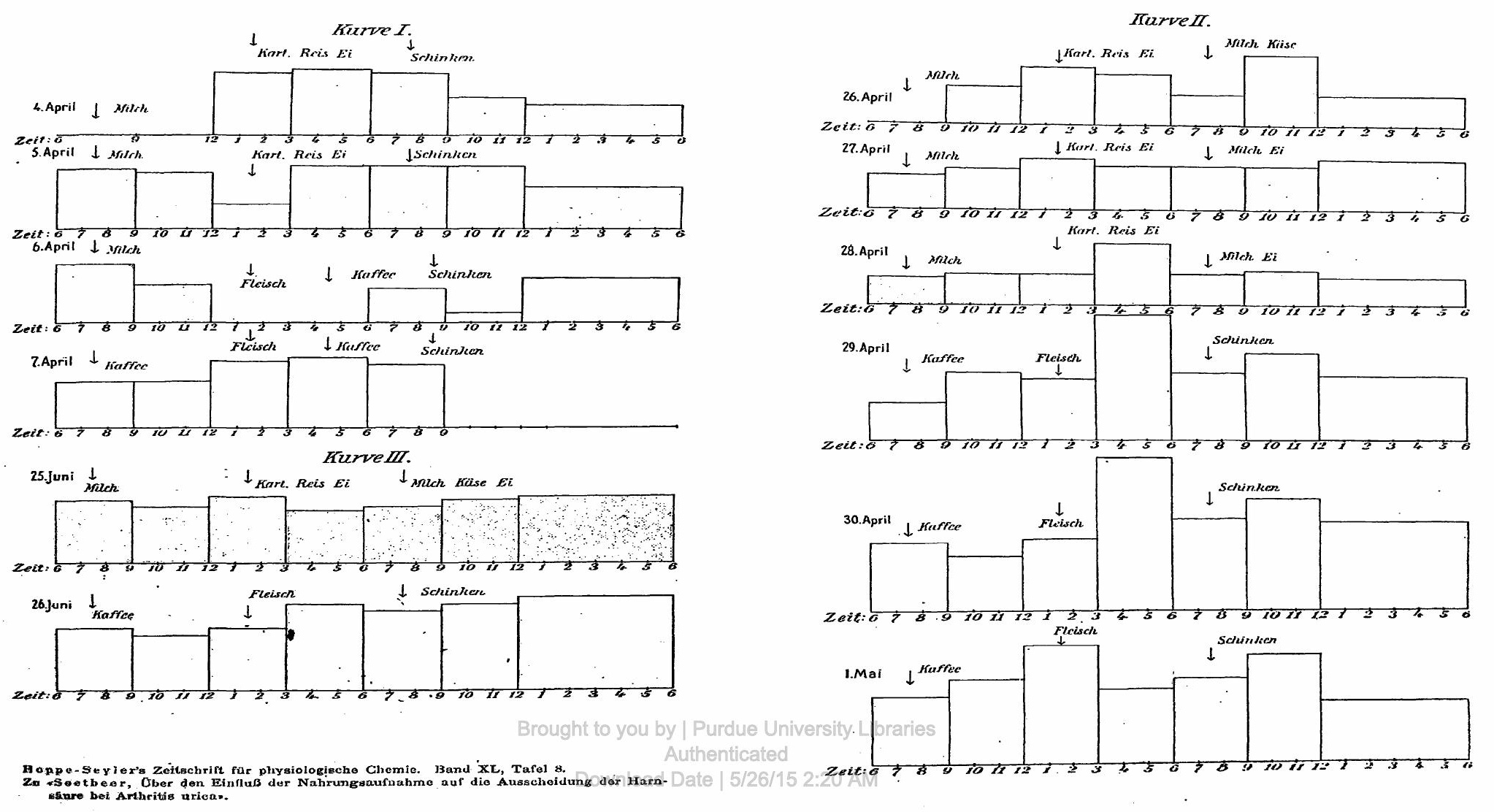


Brought to you by | Purdue University $L$ Authenticated

Download Date | 5/26/15 2:20 Al 


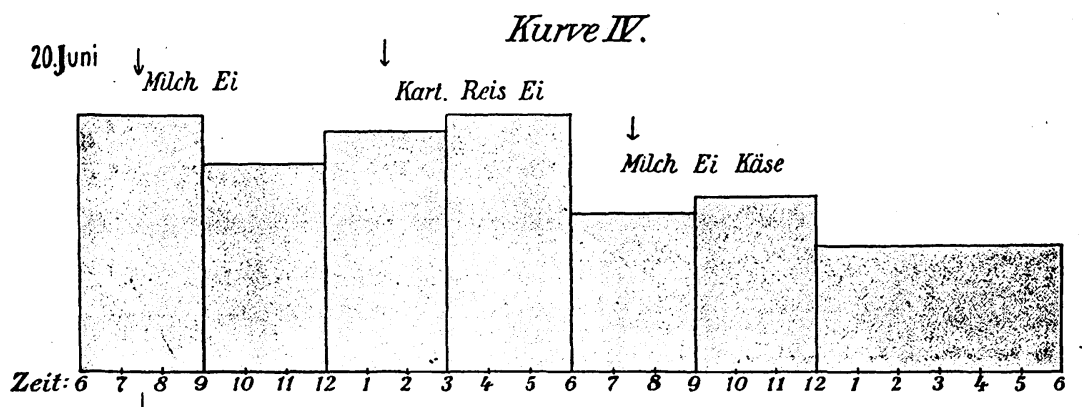
21.Juni $\downarrow$ Milch Ei
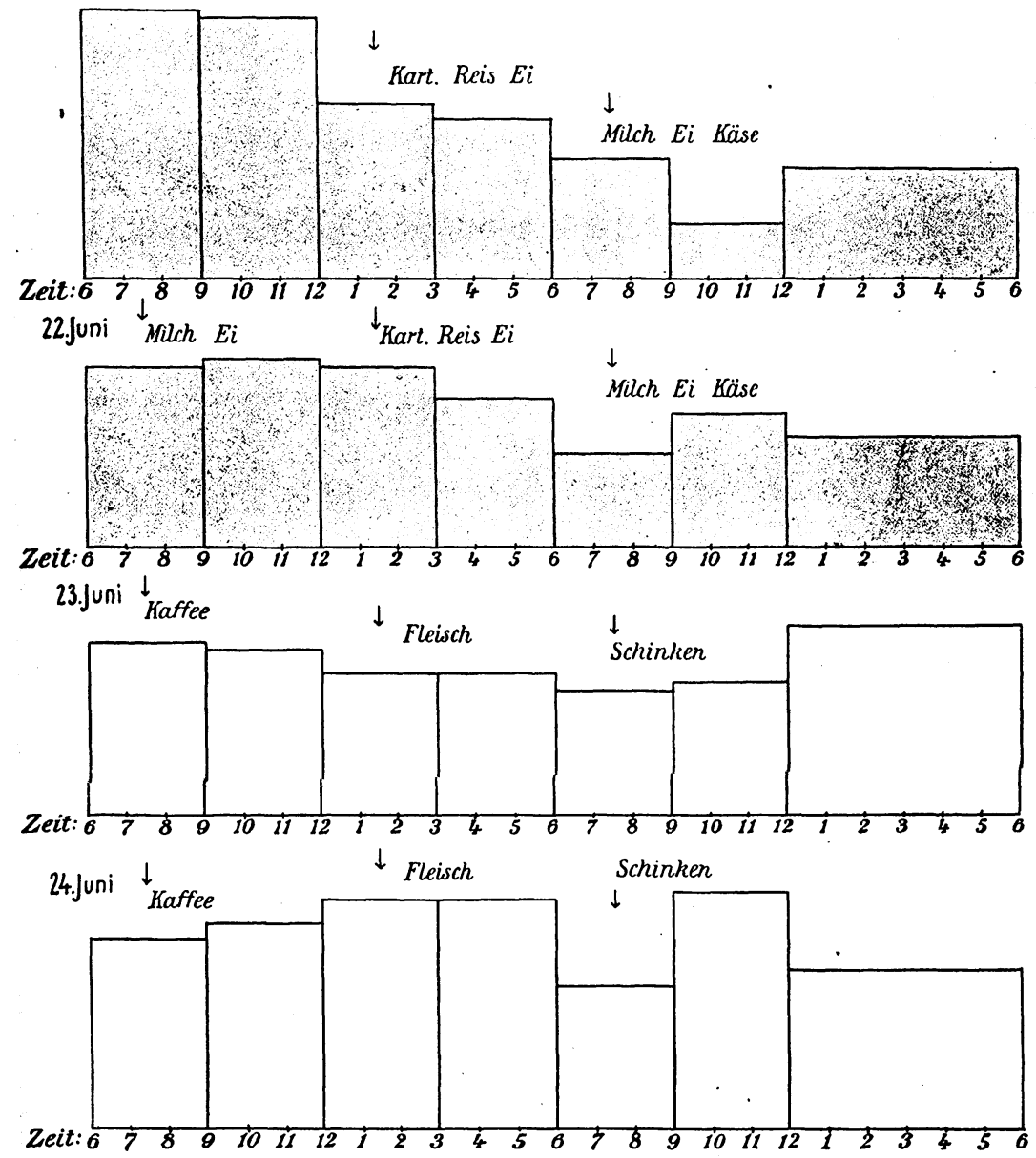

Hoppe-Seyler's Zeitschritt für physiologische Chemie.' Band XL, Tafel 9.

$\mathrm{Zu}$ «Soetbeer, Über den Einfluß der Nahrungsaufnahme auf die Ausscheidung der Harnsäure bei Arthritis urica». 
Brought to you by | Purdue University L Authenticated

Download Date | 5/26/15 2:20 Al 


\section{Kurve $\Gamma$.}
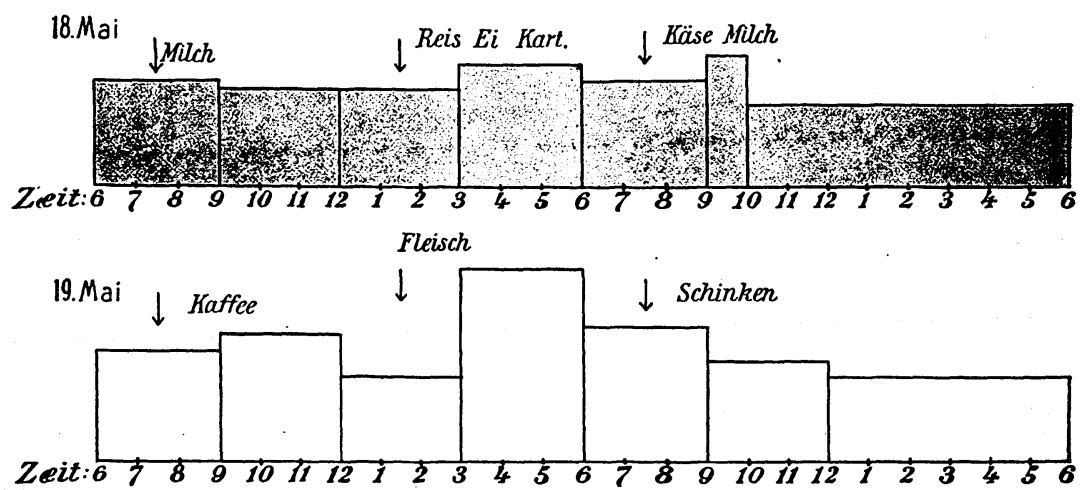

Fleisch
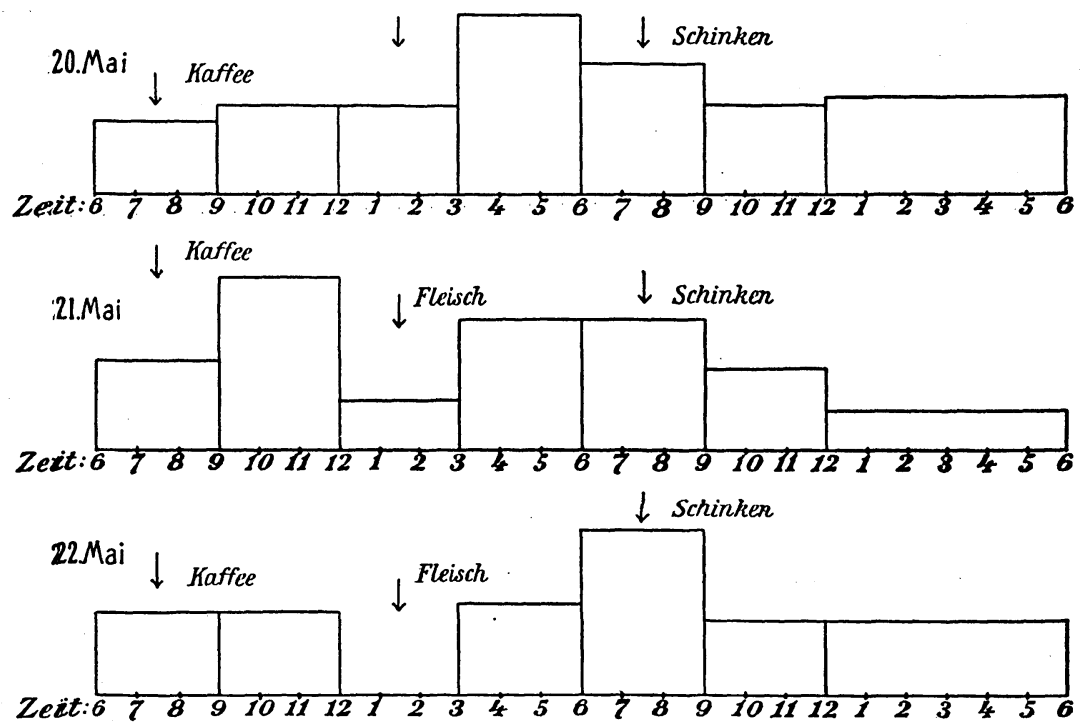

Hoppe-Seyler's Zeitschrift für physiologische Chemie. Band XL, Tafel 10.

$\mathrm{Z} u$ «Soetbeer, Über den Einfluß der Nahrungsaufnahme auf die Ausscheidung der Harnsäure bei Arthritis urica». 
Brought to you by | Purdue University L Authenticated

Download Date | 5/26/15 2:20 Al 